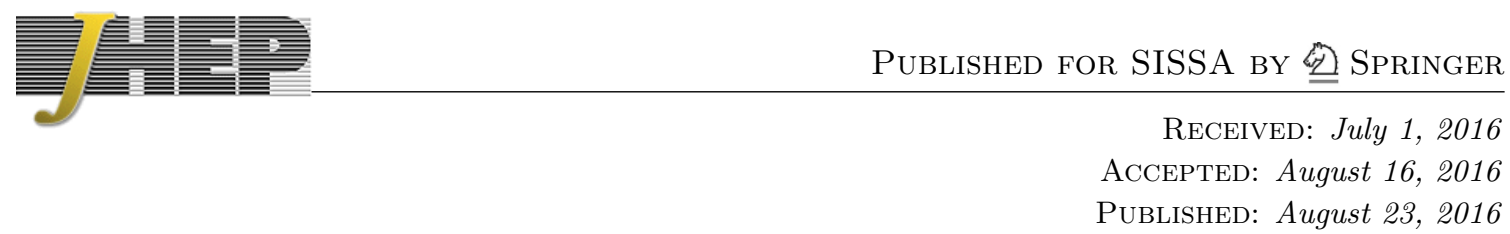

\title{
The SU(2|3) dynamic two-loop form factors
}

\author{
A. Brandhuber, ${ }^{a}$ M. Kostacińska, ${ }^{a}$ B. Penante,${ }^{a, b}$ G. Travaglini ${ }^{a}$ and D. Young ${ }^{a}$ \\ ${ }^{a}$ Centre for Research in String Theory, School of Physics and Astronomy, \\ Queen Mary University of London, \\ Mile End Road, London E1 $4 N S$, U.K. \\ ${ }^{b}$ Institut für Physik und IRIS Adlershof, Humboldt Universität zu Berlin, \\ Zum Großen Windkanal 6, 12489 Berlin, Germany \\ E-mail: a.brandhuber@qmul.ac.uk, m.m.kostacinska@qmul.ac.uk, \\ b.penante@qmul.ac.uk, g.travaglini@qmul.ac.uk, d.young@qmul.ac.uk
}

ABSTRACT: We compute two-loop form factors of operators in the $\mathrm{SU}(2 \mid 3)$ closed subsector of $\mathcal{N}=4$ supersymmetric Yang-Mills. In particular, we focus on the non-protected, dimension-three operators $\operatorname{Tr}(X[Y, Z])$ and $\operatorname{Tr}(\psi \psi)$ for which we compute the four possible two-loop form factors, and corresponding remainder functions, with external states $\langle\bar{X} \bar{Y} \bar{Z}|$ and $\langle\bar{\psi} \bar{\psi}|$. Interestingly, the maximally transcendental part of the two-loop remainder of $\langle\bar{X} \bar{Y} \bar{Z}|\operatorname{Tr}(X[Y, Z])| 0\rangle$ turns out to be identical to that of the corresponding known quantity for the half-BPS operator $\operatorname{Tr}\left(X^{3}\right)$. We also find a surprising connection between the terms subleading in transcendentality and certain a priori unrelated remainder densities introduced in the study of the spin chain Hamiltonian in the SU(2) sector. Next, we use our calculation to resolve the mixing, recovering anomalous dimensions and eigenstates of the dilatation operator in the $\mathrm{SU}(2 \mid 3)$ sector at two loops. We also speculate on potential connections between our calculations in $\mathcal{N}=4$ super Yang-Mills and Higgs + multi-gluon amplitudes in QCD in an effective Lagrangian approach.

KEYWORds: Scattering Amplitudes, Extended Supersymmetry, Supersymmetric gauge theory

ARXIV EPRINT: 1606.08682 


\section{Contents}

1 Introduction 2

2 One-loop minimal form factor $\langle\bar{X} \bar{Y} \bar{Z}|\operatorname{Tr} X[Y, Z]| 0\rangle$

2.1 Setting up the notation 5

2.2 A useful decomposition 6

$\begin{array}{lll}2.3 & \text { Two-particle cuts and result } & 7\end{array}$

2.4 Auxiliary one-loop form factors needed for two-loop cuts 8

3 Two-loop minimal form factor $\langle\bar{X} \bar{Y} \bar{Z}|\operatorname{Tr} X[Y, Z]| 0\rangle \quad 10$

$\begin{array}{lll}3.1 & \text { Two-particle cuts of the two-loop form factor } & 10\end{array}$

$\begin{array}{lll}3.1 .1 & \text { Tree-level form factor } \times \text { one-loop amplitude } & 10\end{array}$

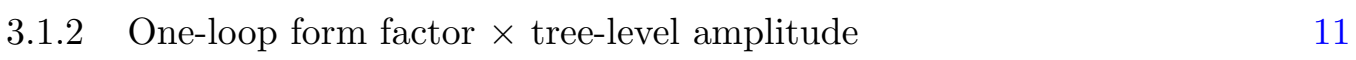

$\begin{array}{lll}3.1 .3 & \text { Result of two-particle cuts } & 14\end{array}$

$\begin{array}{lll}3.2 & \text { Three-particle cuts of the two-loop form factor } & 15\end{array}$

$\begin{array}{lll}3.2 .1 & \text { Three-particle cuts in the } q^{2} \text {-channel } & 15\end{array}$

$\begin{array}{ll}3.2 .2 & \text { Three-particle cuts in the } s_{23} \text {-channel } \\ & 16\end{array}$

$\begin{array}{lll}3.3 & \text { Summary and integral reduction } & 18\end{array}$

4 Two-loop remainder of $\langle\bar{X} \bar{Y} \bar{Z}|\operatorname{Tr} X[Y, Z]| 0\rangle \quad 20$

4.1 Definition of the remainder 20

4.2 A connection to the remainder densities in the $\mathrm{SU}(2)$ sector 23

5 One-loop non-minimal form factor $\left\langle\bar{X} \bar{Y} \bar{Z}\left|\frac{1}{2} \operatorname{Tr} \psi^{\alpha} \psi_{\alpha}\right| 0\right\rangle$

5.1 Two-particle cut in the $q^{2}$-channel 24

$\begin{array}{lll}5.2 & \text { Two-particle cut in the } s_{23} \text {-channel } & 25\end{array}$

$\begin{array}{lll}5.3 & \text { Final result } & 28\end{array}$

6 Two-loop sub-minimal form factor $\langle\bar{\psi} \bar{\psi}|\operatorname{Tr} X[Y, Z]| 0\rangle \quad 29$

7 Two-loop dilatation operator in the $\mathrm{SU}(2 \mid 3)$ sector $\quad 30$

8 Conclusions $\quad 34$

$\begin{array}{ll}\text { A One-loop integral functions } & 35\end{array}$

$\begin{array}{ll}\text { B Comparing half-BPS form factors } & 36\end{array}$ 


\section{Introduction}

The study of form factors of composite operators is a very active area of research. After the pioneering paper [1], interest in the calculation of form factors in supersymmetric theories was rekindled at strong coupling in [2] and at weak coupling in [3]. Specifically, in [3] the study of the simplest possible form factors was undertaken, namely form factors of quadratic half-BPS operators in $\mathcal{N}=4$ supersymmetric Yang-Mills (SYM). The three- and four-loop result for the simplest, two-point (or Sudakov) form factors were then derived in $[4,5]$, respectively. In [6] two-loop form factors of the form $\left\langle\bar{X} \bar{X} g^{ \pm}\left|\operatorname{Tr}\left(X^{2}\right)\right| 0\right\rangle$ were computed, where $X$ is one of the three complex scalar fields of the theory, and $g^{+}\left(g^{-}\right)$denotes a gluon of positive (negative) helicity. In that paper it was also shown that these form factors are identical to the form factors of the self-dual field strength ${ }^{1} F_{\mathrm{SD}},\left\langle g^{+} g^{+} g^{ \pm}\left|\operatorname{Tr}\left(F_{\mathrm{SD}}\right)^{2}\right| 0\right\rangle$ (divided by the corresponding tree-level contribution) thanks to supersymmetric Ward identities $[7,8]$. Indeed, the operators $\operatorname{Tr}\left(X^{2}\right)$ and the on-shell Lagrangian belong to the simplest operator multiplet in the $\mathcal{N}=4$ theory, namely the protected stress-tensor multiplet. Remarkably, in QCD the form factors of $\operatorname{Tr}\left(F^{2}\right)$ compute the leading contribution to Higgs + multi-gluon amplitudes in an effective Lagrangian approach [9-17] in the large top mass limit. The corresponding interaction has the form $\mathcal{L}_{\text {eff }}^{(0)} \sim H \operatorname{Tr}\left(F^{2}\right)$, and hence the quantity ${ }^{2}$

$$
\left\langle g g \cdots g\left|\int d^{4} x e^{-i q \cdot x} \operatorname{Tr}\left(F^{2}\right)(x)\right| 0\right\rangle
$$

precisely computes the amplitude for the process $H \rightarrow g g \cdots g$, with $q^{2}=m_{\mathrm{H}}^{2}$.

Of course, there is no a priori connection between the quantity (1.1) evaluated in $\mathrm{QCD}$ and in $\mathcal{N}=4 \mathrm{SYM}$. Yet, in [6] it was realised that the three-point form factor computed there, $\left\langle\bar{X} \bar{X} g^{+}\left|\operatorname{Tr}\left(X^{2}\right)\right| 0\right\rangle$, is identical to the maximally transcendental part of the amplitudes for $H \rightarrow g^{+} g^{+} g^{ \pm}$calculated in $[18,19]$. This led to the conjecture that the "most complicated part", i.e. the maximally transcendental contribution to Higgs plus multi-gluon processes, at infinite top quark mass, can in fact be computed using $\mathcal{N}=4 \mathrm{SYM}$.

The coupling $\mathcal{L}_{\text {eff }}^{(0)}$ quoted earlier is only the first in an effective Lagrangian description of gluon fusion processes. Subleading corrections have been studied in a number of papers, see e.g. $[20,21]$, where the expansion of the effective Lagrangian is written as

$$
\mathcal{L}_{\text {eff }}=\hat{C}_{0} O_{0}+\frac{1}{m_{\text {top }}^{2}} \sum_{i=1}^{4} \hat{C}_{i} O_{i}+\mathcal{O}\left(\frac{1}{m_{\text {top }}^{4}}\right),
$$

where $O_{i}, i=1, \ldots, 4$ are dimension- 7 operators and $O_{0}=H \operatorname{Tr}\left(F^{2}\right)$.

Some of the operators in the set $\left\{O_{i}\right\}_{i=1}^{4}$ do not contain quarks, and as such can be considered also in $\mathcal{N}=4 \mathrm{SYM}$. In this paper we would like to suggest the relevance of computing form factors of such operators in the maximally supersymmetric theory, and comparing to the QCD results. One possible very interesting scenario is that the $\mathcal{N}=4 \mathrm{SYM}$ calculation continues to capture the maximally transcendental part of the corresponding

\footnotetext{
${ }^{1}$ Or, more precisely, of the on-shell Lagrangian.

${ }^{2}$ Recall that we can separate out $F^{2}=F_{\mathrm{SD}}^{2}+F_{\mathrm{ASD}}^{2}$.
} 
QCD calculation. In particular, the following two operators can be considered,

$$
O_{1}:=H \operatorname{Tr}\left(F^{3}\right)=H\left[\operatorname{Tr}\left(F_{\mathrm{SD}}^{3}\right)+\operatorname{Tr}\left(F_{\mathrm{ASD}}^{3}\right)\right], \quad O_{2}:=H \operatorname{Tr}\left[\left(D^{\mu} F^{\nu \rho}\right)\left(D_{\mu} F_{\nu \rho}\right)\right],
$$

which in QCD are both multiplicatively renormalisable at one loop [22]. Let us briefly discuss the case of $O_{1}$, and in particular the form factor $\left\langle g^{+} g^{+} g^{+}\left|\operatorname{Tr}\left(F^{3}\right)\right| 0\right\rangle$. At tree-level and zero momentum transfer (i.e. $q=0$, where $q$ is the momentum carried by the operator), these form factors become amplitudes produced by higher-dimensional couplings, and have been considered previously in $[13,23,24]$. At $q \neq 0$, they have been studied at tree-level and one loop in [21]. In $\mathcal{N}=4 \mathrm{SYM}$ and at one loop, it turns out that the operator $\operatorname{Tr}\left(F^{3}\right)$ has the same anomalous dimension as the Konishi operator (the calculation of the three-gluon form factor for this operator is currently under investigation). A technically simpler, but equally interesting computation consists of focusing on simpler operators, still containing three fields, and several candidate operators immediately come to mind. The half-BPS operator

$$
\mathcal{O}_{\mathrm{BPS}}=\operatorname{Tr}\left(X^{3}\right),
$$

and its form factors have been studied at one and two loops in [25, 26]. A priori it is however too simple - for instance, unlike $\operatorname{Tr}\left(F^{3}\right)$ in QCD, $\mathcal{O}_{\text {BPS }}$ is protected. Scalar fields are of course preferred, as their form factors are the simplest possible. In order to get a non-protected, trilinear operator we need to consider three complex scalar fields, which we can choose to be

$$
X:=\phi_{12}, \quad Y:=\phi_{23}, \quad Z:=\phi_{31} .
$$

From these fields, one can immediately construct the operators

$$
\begin{aligned}
\tilde{\mathcal{O}}_{\mathrm{BPS}} & :=\operatorname{Tr}(X\{Y, Z\}), \\
\mathcal{O}_{B} & :=\operatorname{Tr}(X[Y, Z]) .
\end{aligned}
$$

While the first operator is another half-BPS combination, ${ }^{3}$ quantum corrections lead to mixing between $\mathcal{O}_{B}$ and the dimension-three operator,

$$
\mathcal{O}_{F}:=\frac{1}{2} \operatorname{Tr}\left(\psi^{\alpha} \psi_{\alpha}\right)
$$

where we have defined

$$
\psi_{\alpha}:=\psi_{123, \alpha} .
$$

The fields $\left\{\phi_{12}, \phi_{23}, \phi_{31} ; \psi_{123, \alpha}\right\}$ are precisely the letters of the $\mathrm{SU}(2 \mid 3)$ closed subsector of $\mathcal{N}=4$ SYM. It has been studied by Beisert in [27, 28], where the dilatation operator was determined up to three loops. Apart from being closed under operator mixing, there is another important feature of this sector: it gives rise to length-changing interactions in the dilatation operator, such as $X Y Z \leftrightarrow \psi \psi$, unlike the (simpler) SU(2) sector.

Motivated by the above discussion, we now describe in more detail the goals of this paper. In the following we will focus on the non-protected, (classically) dimension-three

\footnotetext{
${ }^{3}$ It is symmetric and traceless once written in $\mathrm{SO}(6)$ indices.
} 
operators $\mathcal{O}_{B}$ and $\mathcal{O}_{F}$ for which we compute the four possible two-loop form factors, and corresponding remainder functions with external states $\langle\bar{X} \bar{Y} \bar{Z}|$ and $\langle\bar{\psi} \bar{\psi}|$. It is convenient, and natural from the point of view of operator mixing discussed later, to package them into a matrix of form factors:

$$
\mathcal{F}:=\left(\begin{array}{l}
\left\langle\bar{\psi} \bar{\psi}\left|\mathcal{O}_{F}\right| 0\right\rangle\left\langle\bar{X} \bar{Y} \bar{Z}\left|\mathcal{O}_{F}\right| 0\right\rangle \\
\left\langle\bar{\psi} \bar{\psi}\left|\mathcal{O}_{B}\right| 0\right\rangle\left\langle\bar{X} \bar{Y} \bar{Z}\left|\mathcal{O}_{B}\right| 0\right\rangle
\end{array}\right) .
$$

Apart from the possible connections to phenomenologically relevant quantities in QCD alluded to earlier, there are additional reasons to study form factors of operators such as $\mathcal{O}_{B}$ and $\mathcal{O}_{F}$ :

1. Firstly, it is very interesting to scan the possible remainders of form factors of wider classes of non-protected operators, and compare to results obtained for protected operators and operators belonging to different sectors. A key motivation is to search for regularities and determine universal building blocks in the results that are common to form factors of different operators.

2. By computing loop corrections to minimal form factors of non-protected operators it is possible to find the dilatation operator. This was done recently at one loop for the complete one-loop dilatation operator in [29] and at two loops in the SU(2) subsector [30]. Potentially, this holds promise for gaining further insights into the integrability of $\mathcal{N}=4$ SYM. $^{4}$

The calculation of the two-loop remainder of the form factor $\left\langle\bar{X} \bar{Y} \bar{Z}\left|\mathcal{O}_{B}\right| 0\right\rangle$ is very instructive in this respect. Indeed, we will show that the remainder function is given by a sum of terms of decreasing transcendentality, where the leading, transcendentality-four term turns out to be identical to the remainder for the form factor $\left\langle\bar{X} \bar{X} \bar{X}\left|\operatorname{Tr}\left(X^{3}\right)\right| 0\right\rangle$ computed in [26]. Furthermore, the terms of transcendentality ranging from three to zero turn out to be related to certain finite remainder densities introduced in [30] in the study of the dilatation operator in the $\mathrm{SU}(2)$ sector. It is interesting that they appear (in some form) in the larger $\mathrm{SU}(2 \mid 3)$ sector, possibly pointing to some universality of these quantities. This finding leads us to speculate that the leading transcendental part of the correction terms to Higgs + multi-gluon processes induced by the interactions $O_{i}, i \geq 1$, on the right-hand side of (1.2), can be equivalently obtained by computing their form factors (or form factors related by supersymmetry) in the much simpler $\mathcal{N}=4 \mathrm{SYM}$ theory. The fact that the maximally transcendental part of the form factors in the $\mathrm{SU}(2 \mid 3)$ sector is computed effectively by form factors of half-BPS operators leads us to further speculate on the special role of such operators in computing the maximally transcendental part of the form factors of the operators $O_{i}$ for $i \geq 1$ in QCD.

We will also study and resolve the operator mixing, a problem which requires the knowledge of the ultraviolet (UV) divergences of three additional form factors: $\left\langle\bar{X} \bar{Y} \bar{Z}\left|\mathcal{O}_{F}\right| 0\right\rangle$, $\left\langle\bar{\psi} \bar{\psi}\left|\mathcal{O}_{F}\right| 0\right\rangle$, and $\left\langle\bar{\psi} \bar{\psi}\left|\mathcal{O}_{B}\right| 0\right\rangle$. Note that these four form factors are different in nature: while

\footnotetext{
${ }^{4}$ Complementary approaches based on two-point functions were recently explored in [31-33].
} 
$\left\langle\bar{X} \bar{Y} \bar{Z}\left|\mathcal{O}_{B}\right| 0\right\rangle$ and $\left\langle\bar{\psi} \bar{\psi}\left|\mathcal{O}_{F}\right| 0\right\rangle$ are minimal (i.e. the number of particles in the external state is the same as the number of fields in the operator), $\left\langle\bar{\psi} \bar{\psi}\left|\mathcal{O}_{B}\right| 0\right\rangle$ is sub-minimal (more fields than particles), and $\left\langle\bar{X} \bar{Y} \bar{Z}\left|\mathcal{O}_{F}\right| 0\right\rangle$ is non-minimal. Furthermore, at the loop order we are working the latter two are free from infrared (IR) divergences, lacking a corresponding treelevel form factor. ${ }^{5}$ On the other hand they all have UV divergences, which will be extracted to resolve the mixing and determine the two-loop dilatation operator in the $\mathrm{SU}(2 \mid 3)$ sector, in agreement with [28]. By diagonalising it, two distinguished combinations of $\mathcal{O}_{B}$ and $\mathcal{O}_{F}$ will be determined, one which is half-BPS $[28,35,36]$ and one which is a descendant of the Konishi operator [28, 36-38].

The rest of the paper is organised as follows. In sections 2 and 3 we will derive the form factor $\left\langle\bar{X} \bar{Y} \bar{Z}\left|\mathcal{O}_{B}\right| 0\right\rangle$ at one and two loops, respectively. The two-loop IR-finite (but still UV-divergent) remainder function is then derived in section 4 . There we also establish relations of our result to the results of [26] and [30] for the maximally and subleading transcendental pieces of our result, respectively. In section 5 we compute the sub-minimal form factor $\left\langle\bar{X} \bar{Y} \bar{Z}\left|\mathcal{O}_{F}\right| 0\right\rangle$ up to one loop, which is sufficient for the computation of the two-loop dilatation operator performed later. Section 6 is devoted to computing the subminimal form factor $\left\langle\bar{\psi} \bar{\psi}\left|\mathcal{O}_{B}\right| 0\right\rangle$ at two loops. Using the UV-divergent parts of these form factors, we compute in section 7 the two-loop dilatation operator in the $\mathrm{SU}(2 \mid 3)$ sector, finding its eigenvectors and corresponding anomalous dimensions up to two loops. We conclude with comments on potential future research directions in section 8 .

\section{One-loop minimal form factor $\langle\bar{X} \bar{Y} \bar{Z}|\operatorname{Tr} X[Y, Z]| 0\rangle$}

In this section we consider form factors of the operator introduced in (1.7),

$$
\mathcal{O}_{B}=\operatorname{Tr}(X[Y, Z])
$$

at one loop. Before presenting the calculation we summarise our notation and conventions for the reader's convenience.

\subsection{Setting up the notation}

The fields appearing in the $\mathrm{SU}(2 \mid 3)$ sector are

$$
\left\{X, Y, Z ; \psi_{\alpha}\right\}
$$

previously introduced in (1.5) and (1.9). We recall that the fields $\phi_{A B}$ satisfy the reality condition

$$
\phi^{A B}=\bar{\phi}_{A B}=\frac{1}{2} \epsilon^{A B C D} \phi_{C D}
$$

and therefore

$$
\bar{X}=\phi_{34}=\phi^{12}, \quad \bar{Y}=\phi_{14}=\phi^{23}, \quad \bar{Z}=\phi_{24}=\phi^{31} .
$$

\footnotetext{
${ }^{5}$ We also note that the discontinuities of sub-minimal form factors at two loops were computed in [34] in complete generality.
} 
We also introduce

$$
\psi_{A B C, \alpha}=\epsilon_{A B C D} \psi_{\alpha}^{D}, \quad \bar{\psi}_{\dot{\alpha}}^{A B C}=\epsilon^{A B C D} \bar{\psi}_{D, \dot{\alpha}} .
$$

In our conventions all on-shell particles appearing in amplitudes or form factors are outgoing while the momentum $q$ of the off-shell operator in a form factor is by definition incoming. Therefore it is natural to introduce the Nair super-annihilation operator as

$$
\begin{aligned}
\Phi(p, \eta)= & g^{(+)}(p)+\eta_{A} \psi^{A}(p)+\frac{1}{2} \phi^{A B}(p) \eta_{A} \eta_{B}+\frac{1}{3 !} \bar{\psi}^{A B C}(p) \eta_{A} \eta_{B} \eta_{C} \\
& +g^{(-)}(p) \eta_{1} \cdots \eta_{4},
\end{aligned}
$$

where $g^{(+)}(p), \psi^{A}(p), \phi^{A B}(p), \bar{\psi}^{A B C}(p)$ and $g^{(-)}(p)$, denote the annihilation operators for the various particles of $\mathcal{N}=4 \mathrm{SYM}$. For instance $\langle 0| \psi^{A}(p)$ is a state of an outgoing fermion with momentum $p$ and helicity $+1 / 2$, while $\langle 0| \bar{\psi}^{A B C}(p)$ has momentum $p$ and helicity $-1 / 2$. In the following we will usually denote multiparticle states with on-shell momenta $\left\langle\psi\left(p_{i}\right)^{A_{i}} \cdots \phi\left(p_{j}\right)^{A_{j} B_{j}} \cdots \bar{\psi}\left(p_{k}\right)^{A_{k} B_{k} C_{k}} \cdots\right|$ in the slightly more compact notation

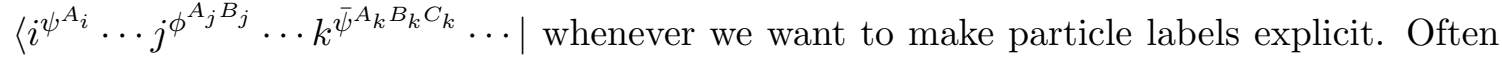
we will also use the following shorthand notation if labels are not needed, in particular $\langle\bar{X} \bar{Y} \bar{Z}|:=\left\langle 1^{\phi^{12}} 2^{\phi^{23}} 3^{\phi^{31}}\right|$ and $\langle\bar{\psi} \bar{\psi}|:=\left\langle 1^{\bar{\psi}^{123}} 2^{\bar{\psi}^{123}}\right|$.

\subsection{A useful decomposition}

In order to compute the form factor $\left\langle\bar{X} \bar{Y} \bar{Z}\left|\mathcal{O}_{B}\right| 0\right\rangle$, with $\mathcal{O}_{B}$ defined in (1.7), we will make use of the decomposition

$$
\mathcal{O}_{B}=\tilde{\mathcal{O}}_{\text {BPS }}+\mathcal{O}_{\text {offset }},
$$

where $\tilde{\mathcal{O}}_{\mathrm{BPS}}$ is the half-BPS operator defined in (1.6) and

$$
\mathcal{O}_{\text {offset }}:=-2 \operatorname{Tr}(X Z Y) \text {. }
$$

This decomposition turns out to be particularly useful for two reasons:

1. Firstly, it separates out the contribution of the half-BPS operator $\tilde{\mathcal{O}}_{\mathrm{BPS}}$. The result for the corresponding half-BPS form factor is identical to that of the half-BPS operator $\operatorname{Tr}\left(X^{3}\right)$ obtained in $[25,26]$ up to two loops and need not be computed again. ${ }^{6}$

2. Secondly, the form factor of the offset operator $\left\langle\bar{X} \bar{Y} \bar{Z}\left|\mathcal{O}_{\text {offset }}\right| 0\right\rangle$ turns out to be particularly simple because of the "shuffled" configuration of the state with respect to the fields inside the operator. ${ }^{7}$ Specifically, we will find that this form factor is expressed in terms of functions with strictly sub-maximal degree of transcendentality, while the half-BPS operator is expressed in terms of functions with maximal degree of transcendentality only.

Therefore we focus on the "offset" operator introduced in (2.7), from which the results for $\mathcal{O}_{B}$ can then be easily obtained.

\footnotetext{
${ }^{6}$ See appendix B for details.

${ }^{7}$ Note that we could have performed the decomposition $\operatorname{Tr}(X[Y, Z])=-\operatorname{Tr}(X\{Y, Z\})+2 \operatorname{Tr}(X Y Z)$ but this is not convenient for our choice of external state $\langle\bar{X} \bar{Y} \bar{Z}|$.
} 


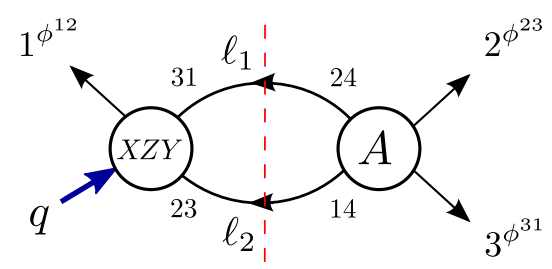

Figure 1. Two-particle cut of the one-loop form factor $F_{\mathcal{O}_{\text {offset }}^{(1)}}\left(1^{\phi^{12}}, 2^{\phi^{23}}, 3^{\phi^{31}} ; q\right)$. We remind the reader of our notation: $X=\phi_{12}, Y=\phi_{23}, Z=\phi_{31}$, with $\bar{X}=\phi^{12}, \bar{Y}=\phi^{23}$ and $\bar{Z}=\phi^{31}$.

\subsection{Two-particle cuts and result}

In the following we denote by $F_{\mathcal{O}_{\text {offset }}}^{(L)}\left(1^{\phi^{12}}, 2^{\phi^{23}}, 3^{\phi^{31}} ; q\right)$ the $L$-loop contribution to the form factor $\left\langle\bar{X} \bar{Y} \bar{Z}\left|\mathcal{O}_{\text {offset }}(0)\right| 0\right\rangle$. We begin by computing $F_{\mathcal{O}_{\text {offset }}}^{(1)}\left(1^{\phi^{12}}, 2^{\phi^{23}}, 3^{\phi^{31}} ; q\right)$ with the twoparticle cut shown in figure 1. This, plus two cyclic permutations of the external particles, are the only cuts contributing to this form factor.

The tree-level amplitude entering the cut is

$$
A\left(2^{\phi^{23}}, 3^{\phi^{31}}, \ell_{2}^{\phi^{14}}, \ell_{1}^{\phi^{24}}\right)=i
$$

while the required tree-level form factor is

$$
F_{\mathcal{O}_{\text {offset }}}^{(0)}\left(1^{\phi^{12}},-\ell_{1}^{\phi^{31}},-\ell_{2}^{\phi^{23}} ; q\right)=-2 .
$$

Hence, uplifting the cut we simply get bubble integrals: ${ }^{8}$

$$
F_{\mathcal{O}_{\text {offset }}}^{(1)}\left(1^{\phi^{12}}, 2^{\phi^{23}}, 3^{\phi^{31}} ; q\right)=2 i \times \nearrow_{1}^{q}+\operatorname{cyclic}(1,2,3) .
$$

A similar calculation shows that, as anticipated, the one-loop form factor of the operator $\tilde{\mathcal{O}}_{\text {BPS }}$ introduced in (1.6) is identical to that of the operator $\operatorname{Tr}\left(X^{3}\right)$ computed in [25],

$$
F_{\tilde{\mathcal{O}}_{\mathrm{BPS}}}^{(1)}\left(1^{\phi^{12}}, 2^{\phi^{23}}, 3^{\phi^{31}} ; q\right)=i s_{23} \times \overbrace{3}^{q}+\operatorname{cyclic}(1,2,3) .
$$

Thus, the one-loop form factor of $\mathcal{O}_{B}$ is ${ }^{9}$

$$
F_{\mathcal{O}_{B}}^{(1)}\left(1^{\phi^{12}}, 2^{\phi^{23}}, 3^{\phi^{31}} ; q\right)=2 i \times \overbrace{1}^{q}+i s_{23} \times \overbrace{3}^{2}+\operatorname{cyclic}(1,2,3),
$$

where $s_{i j}:=\left(p_{i}+p_{j}\right)^{2}$ as usual.

\footnotetext{
${ }^{8}$ Note that each of the cut propagators carries an additional factor of $i$.

${ }^{9}$ Expressions for the one-loop master integrals can be found in appendix A.
} 
From (2.12) we can easily extract the one-loop anomalous dimension of $\mathcal{O}_{B}$. In order to extract the UV divergence from (2.12) we have to remove the IR divergences which is achieved by simply dropping the triangle integrals. Using the results of appendix A, we find the UV divergence at the renormalisation scale $\mu_{R}$ to be

$$
\left.F_{\mathcal{O}_{B}}^{(1)}\right|_{\mu_{R}, \mathrm{UV}}=-\frac{6}{\epsilon} a\left(\mu_{R}\right)
$$

where

$$
a\left(\mu_{R}\right):=\frac{g^{2} N e^{-\epsilon \gamma_{\mathrm{E}}}}{(4 \pi)^{2-\epsilon}}\left(\frac{\mu_{R}}{\mu}\right)^{-2 \epsilon},
$$

and $\mu$ is the usual dimensional regularisation mass parameter. From this we can read off the one-loop anomalous dimension via

$$
\gamma_{\mathcal{O}}=-\left.\mu_{R} \frac{\partial}{\partial \mu_{R}} \log \left(1+\mathcal{Z}_{\mathcal{O}}^{(1)}+\cdots\right)\right|_{\epsilon \rightarrow 0},
$$

with

$$
\mathcal{Z}_{\mathcal{O}_{B}}^{(1)}=\frac{6}{\epsilon} a\left(\mu_{R}\right)
$$

This leads to

$$
\gamma_{\mathcal{O}_{B}}^{(1)}=12 a,
$$

where $a$ is the four-dimensional 't Hooft coupling, given by

$$
a:=\frac{g^{2} N}{(4 \pi)^{2}} .
$$

The result (2.17) is in agreement with known results for the one-loop anomalous dimension of the Konishi multiplet. The same value can be obtained with an explicit application of the formula for the complete one-loop dilatation operator of [34].

\subsection{Auxiliary one-loop form factors needed for two-loop cuts}

In this section we discuss two additional one-loop form factors that will appear as building blocks for the two-particle cuts of the two-loop form factor of $\mathcal{O}_{\text {offset }}$ (and thus $\mathcal{O}_{B}$ ) in section 3.1.2.

The first form factor we consider is $F_{\mathcal{O}_{\text {offset }}}^{(1)}\left(1^{\phi^{12}}, 2^{\phi^{31}}, 3^{\phi^{23}} ; q\right)$, where now the ordering of the particles in the state parallels that of the fields in the operator. A simple two-particle cut is sufficient to determine it, see figure 2.

The amplitude entering the cut is

$$
A\left(2^{\phi^{31}}, 3^{\phi^{23}}, \ell_{2}^{\phi^{14}}, \ell_{1}^{\phi^{24}}\right)=i \frac{\left\langle 2 \ell_{2}\right\rangle\left\langle 3 \ell_{1}\right\rangle}{\left\langle 3 \ell_{2}\right\rangle\left\langle\ell_{1} 2\right\rangle},
$$

thus we get

$$
F_{\mathcal{O}_{\text {offset }}}^{(1)}\left(1^{\phi^{12}}, 2^{\phi^{31}}, 3^{\phi^{23}} ; q\right)=-2 i \times \overbrace{3}^{q}-2 i s_{23} \times \overbrace{3}^{2}+\operatorname{cyclic}(1,2,3) .
$$




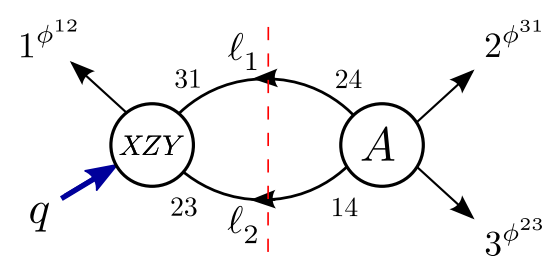

Figure 2. One of the three two-particle cuts of the one-loop form factor $F_{\mathcal{O}_{\text {offset }}}^{(1)}\left(1^{\phi^{12}}, 2^{\phi^{31}}, 3^{\phi^{23}} ; q\right)$. Two more cuts are obtained by cyclically permuting the external legs.

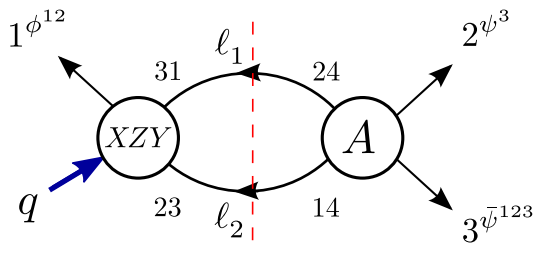

(i)

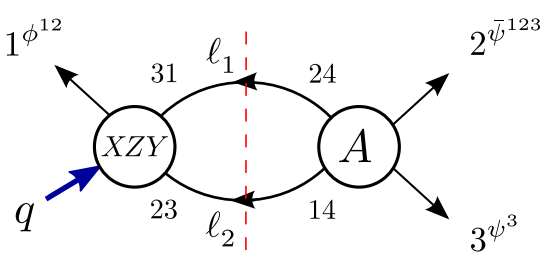

(ii)

Figure 3. One-loop form factors with a fermionic external state entering the two-loop two-particle cuts of figure 6 .

Next we consider the form factors of $\mathcal{O}_{\text {offset }}$ with a fermionic external state made of excitations $\psi^{3}$ and $\bar{\psi}^{123}$, as shown in figure 3 .

The results for the two-particle cuts for the two independent orderings of the fermionic legs are

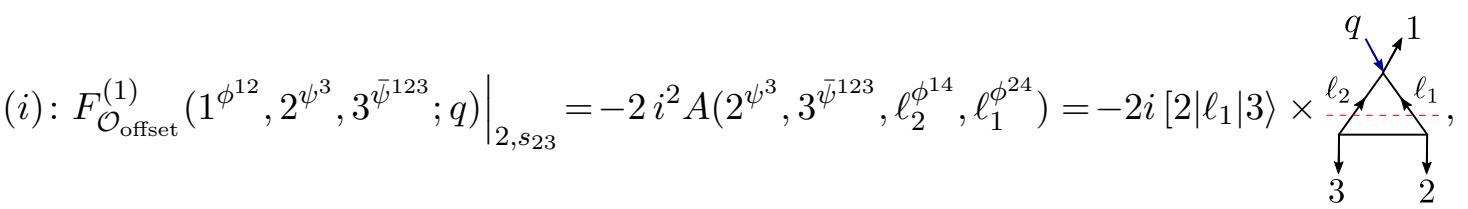

$$
\begin{aligned}
& (i i):\left.F_{\mathcal{O}_{\text {offset }}^{(1)}}\left(1^{\phi^{12}}, 2^{\bar{\psi}^{123}}, 3^{\psi^{3}} ; q\right)\right|_{2, s_{23}}=-2 i^{2} A\left(2^{\bar{\psi}^{123}}, 3^{\psi^{3}}, \ell_{2}^{\phi^{14}}, \ell_{1}^{\phi^{24}}\right)=-2 i\left\langle 2\left|\ell_{2}\right| 3\right] \times \underbrace{\ell_{2}}_{2} \ell_{2}^{\ell_{1}}
\end{aligned}
$$

where we denote the $m$-particle cut of an $L$-loop form factor of an operator $\mathcal{O}$ in a generic $P^{2}$-channel by

$$
\left.F_{\mathcal{O}}^{(L)}(\ldots ; q)\right|_{m, P^{2}}
$$

Both form factors are expressed in terms of a linear triangle which we refrain from reducing to scalar integrals since we are working at the integrand level. ${ }^{10}$ Instead we will plug these expressions into the two-particle cuts of the two-loop form factors shown in figure 6 .

\footnotetext{
${ }^{10}$ Furthermore, both expressions would vanish upon performing the loop integration. Indeed by Lorentz invariance, after Passarino-Veltman reduction one would have e.g. for the first form factor $\ell_{1} \rightarrow a p_{2}+b p_{3}$, thus $\left[2\left|\ell_{1}\right| 3\right\rangle \rightarrow 0$ after the reduction.
} 


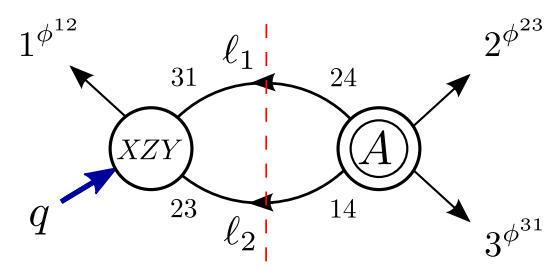

Figure 4. Two-particle cut contributing to the two-loop form factor in the $s_{23}$-channel.

\section{Two-loop minimal form factor $\langle\bar{X} \bar{Y} \bar{Z}|\operatorname{Tr} X[Y, Z]| 0\rangle$}

We proceed to compute the minimal form factor of $\mathcal{O}_{B}=\operatorname{Tr}(X[Y, Z])$ at two loops with the external state $\langle\bar{X} \bar{Y} \bar{Z}|$. The strategy of the calculation is as follows:

1. Thanks to the decomposition (2.6), we need only compute the form factor of the operator $\mathcal{O}_{\text {offset }}=-2 \operatorname{Tr}(X Z Y)$. This will be done in sections 3.1 and 3.2.

2. We then obtain the required form factor of $\mathcal{O}_{B}$ by adding to our result that of the half-BPS operator $\tilde{\mathcal{O}}_{\mathrm{BPS}}=\operatorname{Tr}(X\{Y, Z\})$, which is identical to the form factor $\left\langle\bar{X} \bar{X} \bar{X}\left|\operatorname{Tr}\left(X^{3}\right)\right| 0\right\rangle$ computed in [26], which we quote here for the reader's convenience:

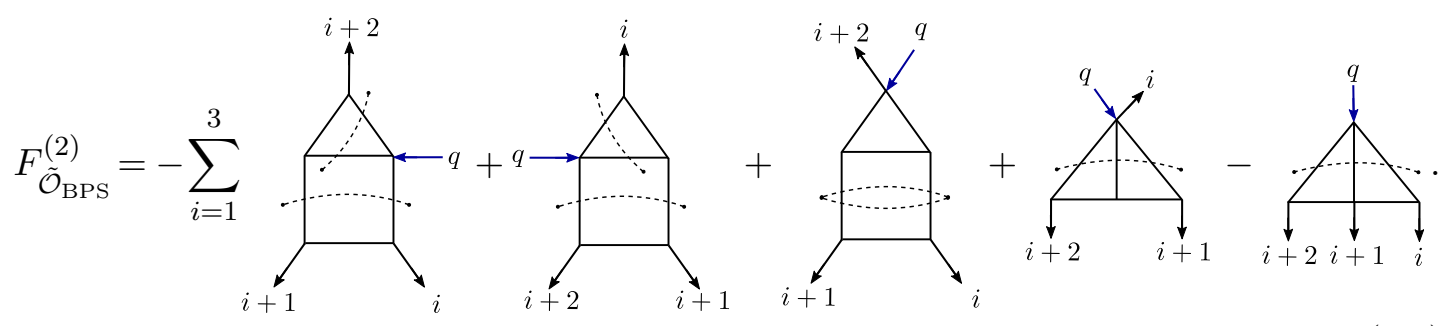

In order to define the numerators we use the notation introduced in [26]: each dashed line corresponds to a numerator factor equal to the total momentum flowing through it, squared. For example, the third integral in (3.1) comes with the factor $\left(s_{i i+1}\right)^{2}$.

3. In section 3.3 we summarise the complete result and perform the integral reduction.

\subsection{Two-particle cuts of the two-loop form factor}

We begin by considering the possible two-particle cuts of the two-loop form factor. There are two types of cuts to consider, which are of the form $F^{(0)} \times A^{(1)}$ and $F^{(1)} \times A^{(0)}$.

\subsubsection{Tree-level form factor $\times$ one-loop amplitude}

The first two-particle cut we consider is of the form $F^{(0)} \times A^{(1)}$, and we will focus on the $s_{23}$-channel. The other cuts are obtained by cyclically permuting the external legs.

In this case the one-loop amplitude is

$$
A^{(1)}=A^{(0)}[-s_{12} s_{23} \times \overbrace{3}^{2}],
$$




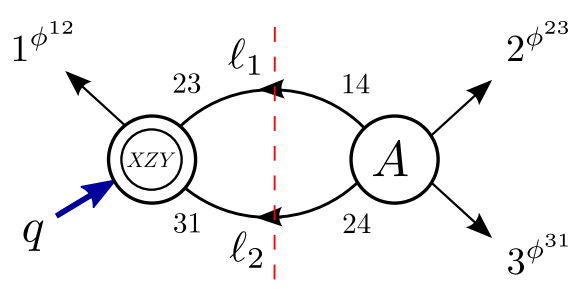

$(i)$

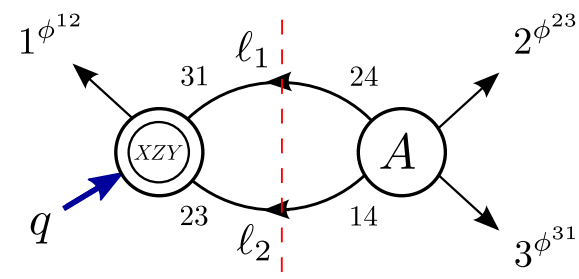

(ii)

Figure 5. Contribution to the two-loop form factor from scalars in the loop.

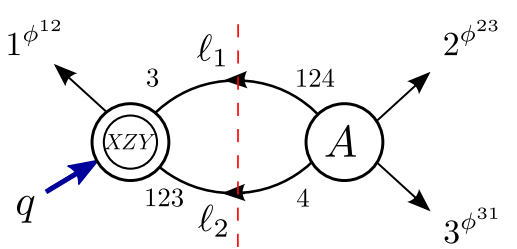

(i)

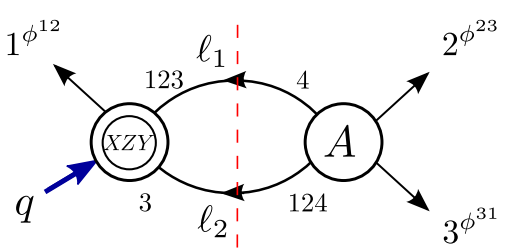

(ii)

Figure 6. Two-loop form factors with internal fermions. The one-loop form factors on the left-hand-side of the cuts were computed in (2.21).

hence the algebra of the previous section iterates and we get the following result for the cut:

$$
\left.F_{\mathcal{O}_{\text {offset }}}^{(2)}\left(1^{\phi^{12}}, 2^{\phi^{23}}, 3^{\phi^{31}} ; q\right)\right|_{2, s_{23}}=-2 s_{23} s_{2 \ell_{1}} \times \overbrace{2}^{\ell_{2}}
$$

\subsubsection{One-loop form factor $\times$ tree-level amplitude}

Next we consider two-particle cuts of the form $F^{(1)} \times A^{(0)}$. There are two options for the states running in the loop: we can either have scalars, as shown in figure 5 , or fermions, as in figure 6. We consider these two types of contributions in turn.

Scalars in the loop. This case is illustrated in figure 5. The relevant one-loop form factors were calculated in section 2.3, while the tree amplitudes entering the cuts are

$$
\begin{aligned}
& \text { (i) : } \quad A\left(2^{\phi^{23}}, 3^{\phi^{31}}, \ell_{2}^{\phi^{24}}, \ell_{1}^{\phi^{14}}\right)=i \frac{\left\langle 2 \ell_{2}\right\rangle\left\langle 3 \ell_{1}\right\rangle}{\left\langle 3 \ell_{2}\right\rangle\left\langle\ell_{1} 2\right\rangle}=-i\left(1+\frac{s_{23}}{2\left(\ell_{1} \cdot p_{2}\right)}\right), \\
& \text { (ii) : } \quad A\left(2^{\phi^{23}}, 3^{\phi^{31}}, \ell_{2}^{\phi^{14}}, \ell_{1}^{\phi^{24}}\right)=i
\end{aligned}
$$


This results in the following possibilities:

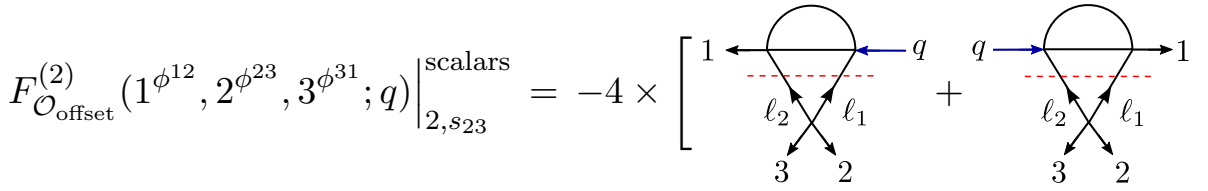

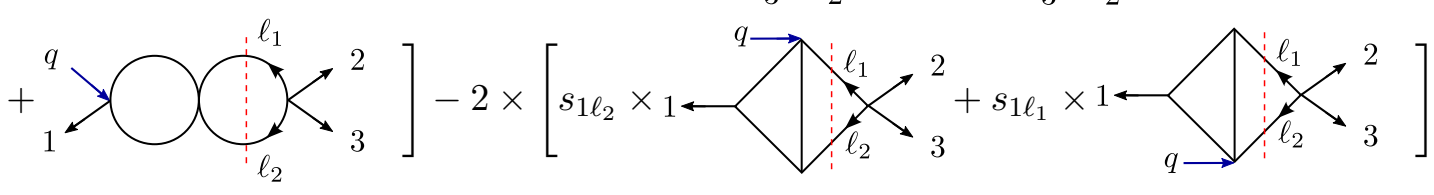

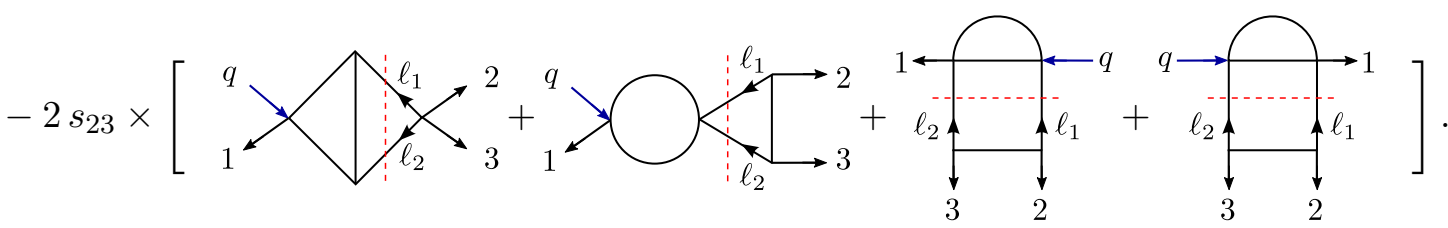

Note that all the topologies which have a one-loop sub-amplitude containing triangles or bubbles have to cancel as a consequence of the amplitude no-triangle theorem [39] — these are the integrals number 3, 6 and 7 in (3.5). This cancellation occurs after adding the contribution from fermions running in the loop, which we compute now.

Fermions in the loop. The contribution from fermions in the loop are shown in figure 6 .

We use the expressions for the one-loop form factors given in $(2.21)^{11}$ and amplitudes, graphically represented as:

$$
\begin{aligned}
& F_{\mathcal{O}_{\text {offset }}}^{(1)}\left(1^{\phi^{12}},-\ell_{1}^{\psi^{3}},-\ell_{2}^{\bar{\psi}^{123}} ; q\right)=2 i\left[\ell_{1}\left|\ell_{4}\right| \ell_{2}\right\rangle \times \overbrace{\ell_{2}}^{\ell_{3}} \ell_{\ell_{1}}^{\ell_{4}}, \\
& F_{\mathcal{O}_{\text {offset }}}^{(1)}\left(1^{\phi^{12}},-\ell_{1}^{\bar{\psi}^{123}},-\ell_{2}^{\psi^{3}} ; q\right)=2 i\left\langle\ell_{1}\left|\ell_{3}\right| \ell_{2}\right] \times \overbrace{\ell_{2}}^{\ell_{3}} \ell_{\ell_{1}}^{\ell_{4}},
\end{aligned}
$$

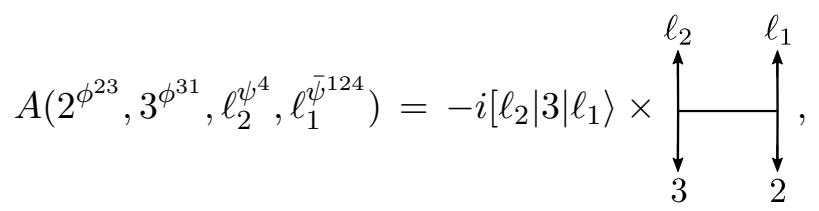

$$
\begin{aligned}
& A\left(2^{\phi^{23}}, 3^{\phi^{31}}, \ell_{2}^{\bar{\psi}^{124}}, \ell_{1}^{\psi^{4}}\right)=-i\left\langle\ell_{2}|2| \ell_{1}\right] \times{\stackrel{\downarrow}{\ell_{2}}}_{\underbrace{}_{2}}^{\ell_{2}^{\ell_{1}}} .
\end{aligned}
$$

\footnotetext{
${ }^{11}$ We added an extra minus sign to every expression to take into account the reversal of direction of $\ell_{1}$ and $\ell_{2}$ according to the following practical prescription [40]: $\lambda_{-P}=-\lambda_{P}, \tilde{\lambda}_{-P}=\tilde{\lambda}_{P}, \eta_{-P}=\eta_{P}$.
} 
We obtain the following results for the cuts shown in figure 6 :

$$
\begin{aligned}
& (i): \quad-i^{2} F_{\mathcal{O}_{\text {offset }}^{(1)}}\left(1^{\phi^{12}},-\ell_{1}^{\psi^{3}},-\ell_{2}^{\bar{\psi}^{123}} ; q\right) \times A\left(2^{\phi^{23}}, 3^{\phi^{31}}, \ell_{2}^{\psi^{4}}, \ell_{1}^{\bar{\psi}^{124}}\right) \\
& =2\left[\ell_{1}\left|\ell_{4}\right| \ell_{2}\right\rangle\left[\ell_{2}|3| \ell_{1}\right\rangle \times \\
& (i i): \quad-i^{2} F_{\mathcal{O}_{\text {offset }}^{(1)}}\left(1^{\phi^{12}},-\ell_{1}^{\bar{\psi}^{123}},-\ell_{2}^{\psi^{3}} ; q\right) \times A\left(2^{\phi^{23}}, 3^{\phi^{31}}, \ell_{2}^{\bar{\psi}^{124}}, \ell_{1}^{\psi^{4}}\right) \\
& =2\left\langle\ell_{1}\left|\ell_{3}\right| \ell_{2}\right]\left\langle\ell_{2}|2| \ell_{1}\right] \times
\end{aligned}
$$

where for convenience we have labeled the additional internal momenta as $k$ and $h$, and we have also multiplied the result of the cut by $(-1)$ from the fermion loop. Note that $\ell_{1}$ and $\ell_{2}$ are cut, while $\ell_{3}, \ell_{4}, k$ and $h$ are off shell.

Combining (3.10) and (3.11) we obtain

$$
[(3.10)+(3.11)]=2\left[\operatorname{Tr}_{+}\left(2 \ell_{1} \ell_{4} \ell_{2}\right)+\operatorname{Tr}_{+}\left(2 \ell_{2} \ell_{4} \ell_{1}\right)\right]
$$

where we used momentum conservation $\ell_{1}+\ell_{2}=\ell_{3}+\ell_{4}=-p_{2}-p_{3}$ and the fact that on the cut $s_{2 \ell_{1}}=s_{3 \ell_{2}}$. Next we evaluate the traces in (3.12) and expand the various scalar products in terms of the inverse propagators appearing in the main topology above, specifically using

$$
\begin{aligned}
& 2\left(\ell_{2} \cdot \ell_{3}\right)=2\left(\ell_{1} \cdot \ell_{4}\right)+\ell_{3}^{2}-\ell_{4}^{2}=-h^{2}+\ell_{3}^{2}, \\
& 2\left(\ell_{4} \cdot \ell_{2}\right)=s_{23}+h^{2}-\ell_{3}^{2}, \\
& 2\left(p_{2} \cdot \ell_{2}\right)=-2\left(p_{2} \cdot \ell_{1}\right)-s_{23}=-k^{2}-s_{23},
\end{aligned}
$$

where $k^{2}=\left(p_{2}+\ell_{1}\right)^{2}=2\left(p_{2} \cdot \ell_{1}\right)$ and $h^{2}=\left(\ell_{1}-\ell_{4}\right)^{2}=-2\left(\ell_{1} \cdot \ell_{4}\right)+\ell_{4}^{2}$. Doing so, we can rewrite (3.12) and obtain the fermionic contribution to the two-particle cut of the two-loop 
form factors of $\mathcal{O}_{\text {offset }}$,

$$
\left.F_{\mathcal{O}_{\text {offset }}}^{(2)}\left(1^{\phi^{12}}, 2^{\phi^{23}}, 3^{\phi^{31}} ; q\right)\right|_{2, s_{23}} ^{\text {fermions }}=2\left[2 k^{2} h^{2}+s_{23}\left(k^{2}+h^{2}\right)-k^{2}\left(\ell_{3}^{2}+\ell_{4}^{2}\right)-s_{23} s_{2 \ell_{4}}\right] \times
$$

From (3.14) we can now proceed to work out the cut integrals contributing to the form factor of $\mathcal{O}_{\text {offset }}$. We arrive at the result

$$
\begin{aligned}
& \left.F_{\mathcal{O}_{\text {offset }}}^{(2)}\left(1^{\phi^{12}}, 2^{\phi^{23}}, 3^{\phi^{31}} ; q\right)\right|_{2, s_{23}} ^{\text {fermions }}=2 s_{23} \times\left[{ }_{1}^{q}\right. \\
& -2 \times\left[s_{23} s_{3 \ell} \times{ }_{3}^{q}\right.
\end{aligned}
$$

We observe that the first, second and last integral in (3.15) precisely cancel the unwanted contributions in (3.5).

\subsubsection{Result of two-particle cuts}

It remains to sum up the scalar and fermion contributions to the cut in question, given in (3.5) and (3.15), respectively. The combined result is:

$$
\text { (2) }\left.\left(1 \phi^{\phi^{2}}, 2^{\phi^{23}}, 3^{\phi^{31}} ; q\right)\right|_{2, s_{23}}
$$


Note that the unwanted topologies which would lead to a violation of the amplitude notriangle theorem [39] have cancelled, as expected. We also observe that some of the numerators in (3.16) are ambiguous due to the cut conditions, and will be determined from three-particle cuts.

\subsection{Three-particle cuts of the two-loop form factor}

In this section we study the three-particle cuts of the form factor of the operator $\mathcal{O}_{\text {offset }}$ defined in (2.7) at two loops. This computation will allow us to fix ambiguities of the numerators of integrals obtained from two-particle cuts and, in addition, provide additional integrals which are not detected by two-particle cuts. We consider three-particle cuts in the $q^{2}$-channel in section 3.2.1, and in the $s_{23}$-channel in section 3.2.2.

\subsubsection{Three-particle cuts in the $q^{2}$-channel}

We begin by studying the three independent $q^{2}$-channel cuts shown in figure 7 .

The corresponding six-point scalar amplitudes are:

$$
\begin{aligned}
& A\left(1^{\phi^{12}}, 2^{\phi^{23}}, 3^{\phi^{31}}, 4^{\phi^{14}}, 5^{\phi^{24}}, 6^{\phi^{34}}\right)=i\left[\frac{1}{s_{126}}+\frac{1}{s_{234}}-\frac{1}{s_{16}}+\frac{s_{12}}{s_{16} s_{126}}+\frac{s_{56}}{s_{16} s_{234}}\right], \\
& A\left(1^{\phi^{12}}, 2^{\phi^{23}}, 3^{\phi^{31}}, 4^{\phi^{24}}, 5^{\phi^{34}}, 6^{\phi^{14}}\right)=i\left[\frac{1}{s_{126}}+\frac{1}{s_{234}}-\frac{1}{s_{34}}+\frac{s_{23}}{s_{34} s_{234}}+\frac{s_{45}}{s_{34} s_{126}}\right], \\
& A\left(1^{\phi^{12}}, 2^{\phi^{23}}, 3^{\phi^{31}}, 4^{\phi^{34}}, 5^{\phi^{14}}, 6^{\phi^{24}}\right)=0
\end{aligned}
$$

where to simplify the notation we have called the cut legs $p_{4}, p_{5}$ and $p_{6}$. We can now immediately read off the contributions to the three-particle cuts: ${ }^{12}$

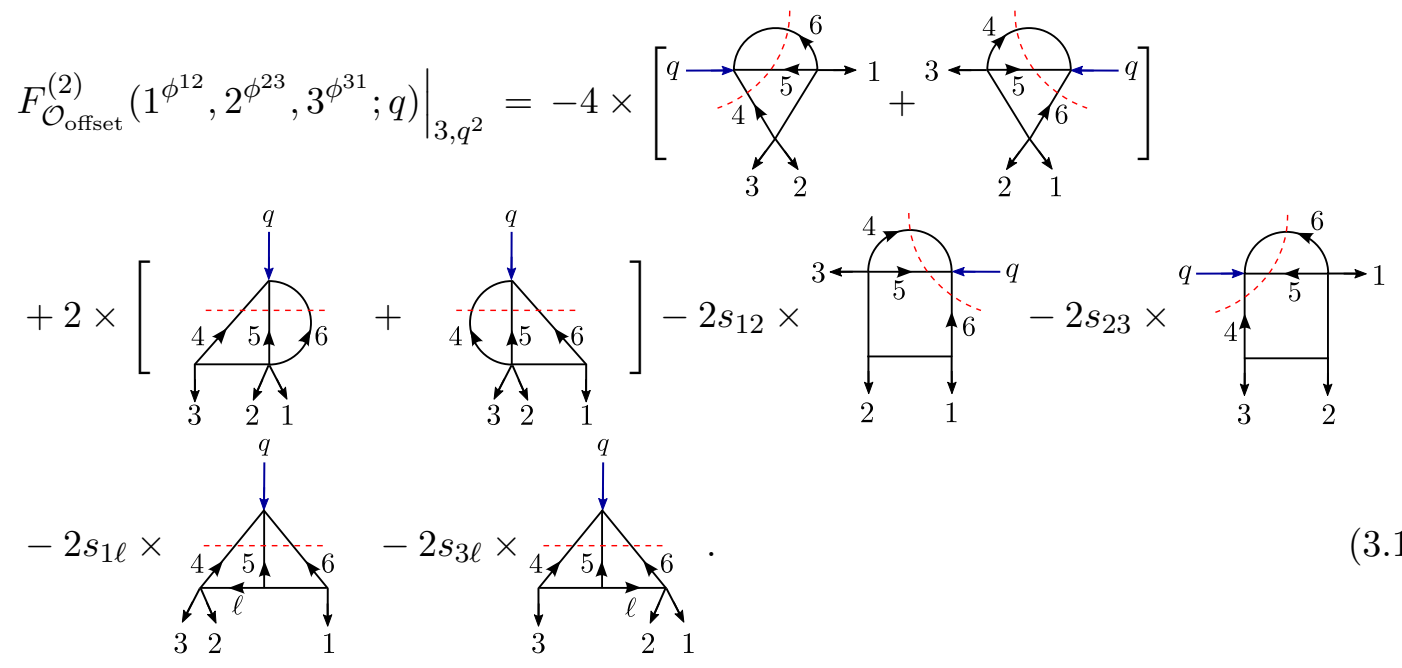

Two observations are in order. Firstly, new topologies have appeared, which do not have two-particle cuts. Furthermore, the ambiguities we had found in some of the numerators of topologies identified using two-particle cuts have now been resolved.

\footnotetext{
${ }^{12}$ Recall that $\mathcal{O}_{\text {offset }}=-2 \operatorname{Tr}(X Z Y)$, and note that in the cuts the factor of $i$ from the amplitude cancels with the factor of $i^{3}$ from the three propagators.
} 


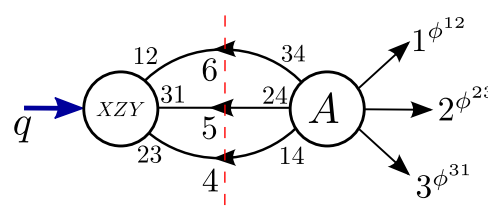

$(i)$

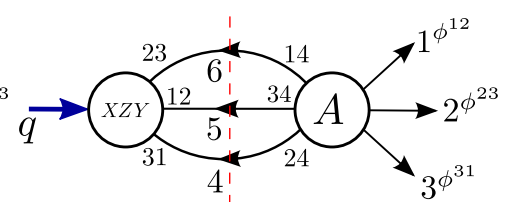

(ii)

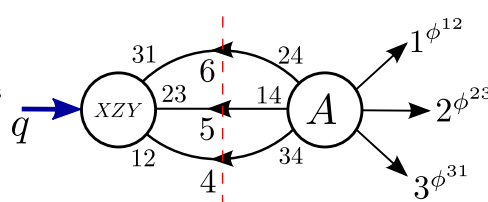

(iii)

Figure 7. Three-particle cuts in the the $q^{2}$-channel.

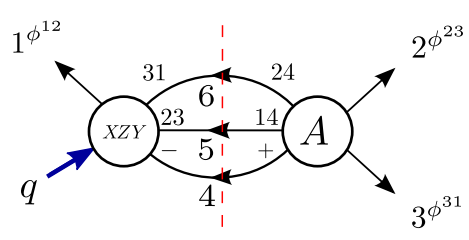

(i)

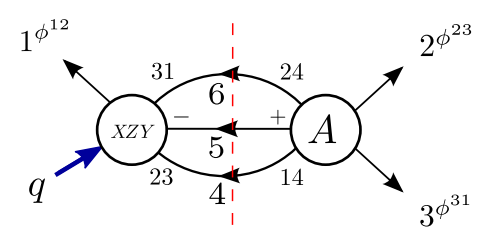

(ii)

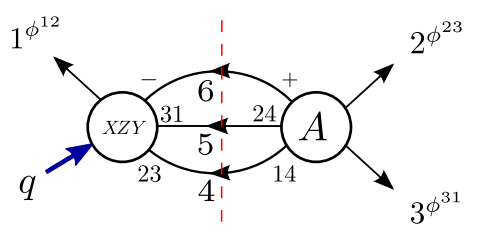

(iii)

Figure 8. Three cut diagrams for the case of a single gluon running in one of the internal loop legs. There are three more diagrams where the internal gluon has the opposite helicity. These are obtained by parity conjugation of the diagrams in this figure.

As a final set of consistency checks, we now perform additional three-particle cuts in the $s_{23}$-channel.

\subsubsection{Three-particle cuts in the $s_{23}$-channel}

In this cut, $R$-symmetry allows for two possibilities for the particles running in the loop, namely two scalars and a gluon, or two fermions and a scalar. There are two distinct situations to consider, namely

$$
F^{\overline{\mathrm{MHV}}} \times A^{\mathrm{MHV}} \quad \text { and } \quad F^{\mathrm{MHV}} \times A^{\overline{\mathrm{MHV}}} .
$$

We now study the first case in detail, while the second can be obtained by just interchanging $\langle\cdot, \cdot\rangle \leftrightarrow[\cdot, \cdot]$ and simply doubles up the contribution from the first case. As before, we focus our attention on the operator $\mathcal{O}_{\text {offset }}$ introduced in (2.7).

Gluons in the loop. The gluon can be exchanged in any of the three loop legs, as shown in figure 8.

The corresponding integrands are

$$
\begin{gathered}
(i): i^{3} A\left(2^{\phi^{23}}, 3^{\phi^{31}}, 4^{+}, 5^{\phi^{14}}, 6^{\phi^{24}}\right) \times F_{\mathcal{O}_{\text {offset }}}^{(0)}\left(1^{\phi^{12}},-6^{\phi^{31}},-5^{\phi^{23}},-4^{-} ; q\right)=\frac{2\langle 35\rangle[51]}{\langle 34\rangle\langle 45\rangle[54][41]}, \\
(i i): i^{3} A\left(2^{\phi^{23}}, 3^{\phi^{31}}, 4^{\phi^{14}}, 5^{+}, 6^{\phi^{24}}\right) \times F_{\mathcal{O}_{\text {offset }}^{(0)}}\left(1^{\phi^{12}},-6^{\phi^{31}},-5^{-},-4^{\phi^{23}} ; q\right)=\frac{2\langle 46\rangle[64]}{\langle 45\rangle\langle 56\rangle[54][65]}, \\
(i i i): i^{3} A\left(2^{\phi^{23}}, 3^{\phi^{31}}, 4^{\phi^{14}}, 5^{\phi^{24}}, 6^{+}\right) \times F_{\mathcal{O}_{\text {offset }}^{(0)}}\left(1^{\phi^{12}},-6^{-},-5^{\phi^{31}},-4^{\phi^{23}} ; q\right)=\frac{2\langle 25\rangle[51]}{\langle 56\rangle\langle 62\rangle[16][65]}
\end{gathered}
$$




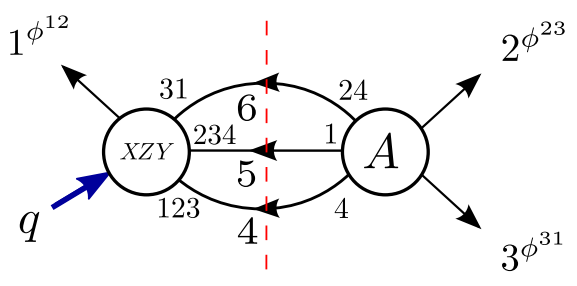

(i)

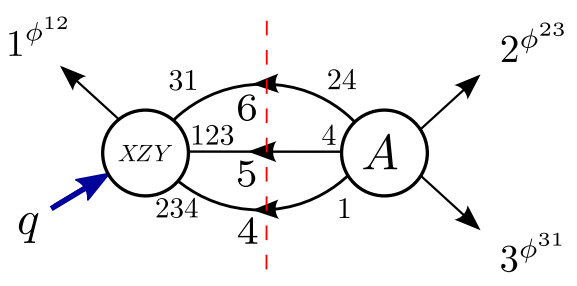

(ii)

Figure 9. The first two diagrams with fermions in the loop. In our conventions, the Yukawa couplings are of the form, schematically, $\operatorname{Tr}\left(\phi^{A B} \bar{\psi}_{A} \bar{\psi}_{B}\right)$ and $\operatorname{Tr}\left(\phi_{A B} \psi^{A} \psi^{B}\right)$, where $\phi_{A B}$ is related to $\phi^{A B}$ via $(2.2)$.

As explained earlier, the three cases corresponding to the opposite helicity assignment of the gluon, which corresponds to $F^{\mathrm{MHV}} \times A^{\overline{\mathrm{MHV}}}$ are related to those discussed above, $F^{\overline{\mathrm{MHV}}} \times A^{\mathrm{MHV}}$, by parity conjugation. The corresponding result is obtained upon interchanging $\langle\cdot, \cdot\rangle \leftrightarrow[\cdot, \cdot]$.

Fermions in the loop. Next we consider the situation where two of the loop legs are fermionic. There are four diagrams corresponding to $F^{\overline{\mathrm{MHV}}} \times A^{\mathrm{MHV}}$, shown in figures 9 and 10. The integrands corresponding to the cuts in figure 9 are

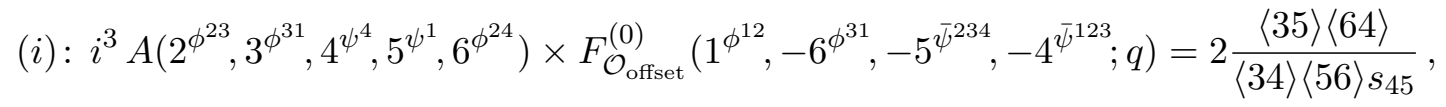

(ii) : $i^{3} A\left(2^{\phi^{23}}, 3^{\phi^{31}}, 4^{\psi^{1}}, 5^{\psi^{4}}, 6^{\phi^{24}}\right) \times F_{\mathcal{O}_{\text {offset }}}^{(0)}\left(1^{\phi^{12}},-6^{\phi^{31}},-5^{\bar{\psi}^{123}},-4^{\bar{\psi}^{234}} ; q\right)=-\frac{2}{s_{45}}$,

while for the cuts in figure 10 we get

$$
\begin{aligned}
& (\text { iii }): i^{3} A\left(2^{\phi^{23}}, 3^{\phi^{31}}, 4^{\phi^{14}}, 5^{\psi^{4}}, 6^{\psi^{2}}\right) \times F_{\mathcal{O}_{\text {offset }}^{(0)}}\left(1^{\phi^{12}},-6^{\bar{\psi}^{134}},-5^{\bar{\psi}^{123}},-4^{\phi^{23}} ; q\right)=-\frac{2}{s_{56}}, \quad(3.25) \\
& (i v): i^{3} A\left(2^{\phi^{23}}, 3^{\phi^{31}}, 4^{\phi^{14}}, 5^{\psi^{2}}, 6^{\psi^{4}}\right) \times F_{\mathcal{O}_{\text {offset }}}^{(0)}\left(1^{\phi^{12}},-6^{\bar{\psi}^{123}},-5^{\bar{\psi}^{134}},-4^{\phi^{23}} ; q\right)=\frac{2}{s_{56}} \frac{\langle 25\rangle\langle 46\rangle}{\langle 45\rangle\langle 62\rangle} .
\end{aligned}
$$

Again, there are four more diagrams corresponding to $F^{\mathrm{MHV}} \times A^{\overline{\mathrm{MHV}}}$ which can be obtained using parity conjugation.

Combining the terms. We can now convert the integrands into traces and dot products and expand them. In doing so, it is useful to notice that the following combination of integrands is particularly simple:

$$
(3.20)+(3.23)+(3.24)+\frac{1}{2}(3.21)=\frac{s_{1 \ell}}{s_{45} s_{14}}+\frac{s_{13}}{s_{34} s_{14}}-\frac{1}{s_{45}}-\frac{s_{23} s_{26}}{s_{34} s_{45} s_{56}}-\frac{1}{s_{14}},
$$

where $\ell=-p_{4}-p_{5}$ and $p_{4}, p_{5}$ and $p_{6}$ are the cut loop momenta. The corresponding integrals are shown in (3.28) below. In uplifting the cut expression, we have to pay close 


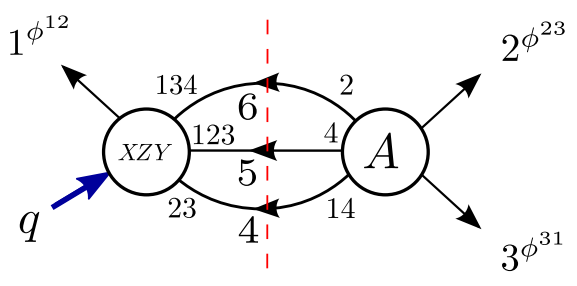

(iii)

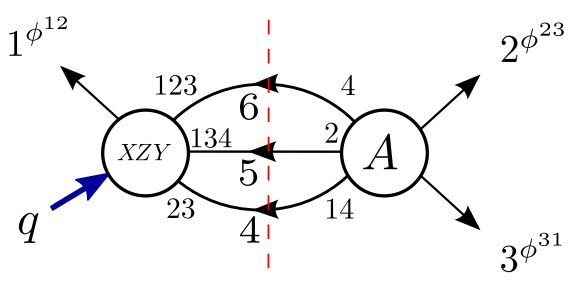

$(i v)$

Figure 10. The remaining two diagrams with fermions in the loop.

attention to the momentum flow: for example, in the expression above $1 / s_{14}=1 /\left[2\left(p_{1} \cdot p_{4}\right)\right]$ should be uplifted to the propagator $-1 /\left(p_{1}-p_{4}\right)^{2}$ since $p_{1}$ and $p_{4}$ flow in the same direction (see figure 9). Keeping these additional signs in mind we arrive at the the following list of integrals:

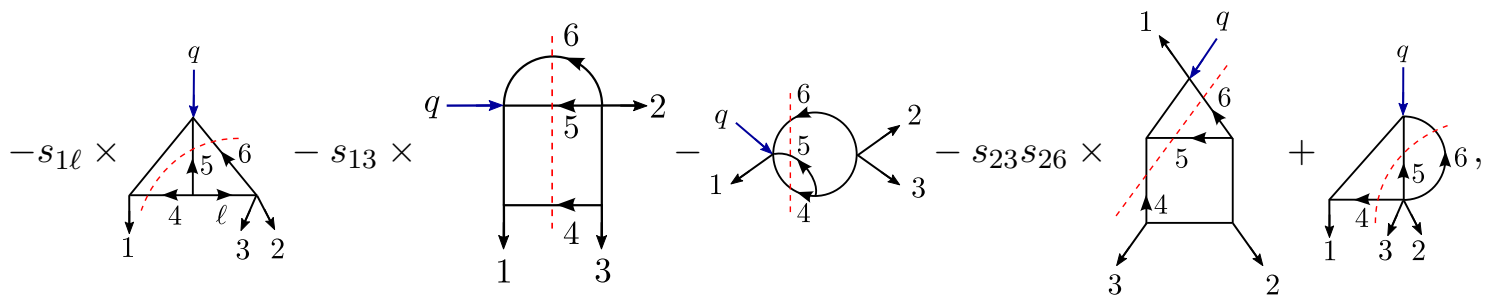

Similarly, we single out the following combination

$$
(3.22)+(3.25)+(3.26)+\frac{1}{2}(3.21)=\frac{s_{1 \ell}}{s_{56} s_{16}}+\frac{s_{12}}{s_{16} s_{26}}-\frac{1}{s_{56}}-\frac{s_{23} s_{34}}{s_{45} s_{56} s_{26}}-\frac{1}{s_{16}},
$$

where $\ell=-p_{5}-p_{6}$. This leads to the integrals shown below,

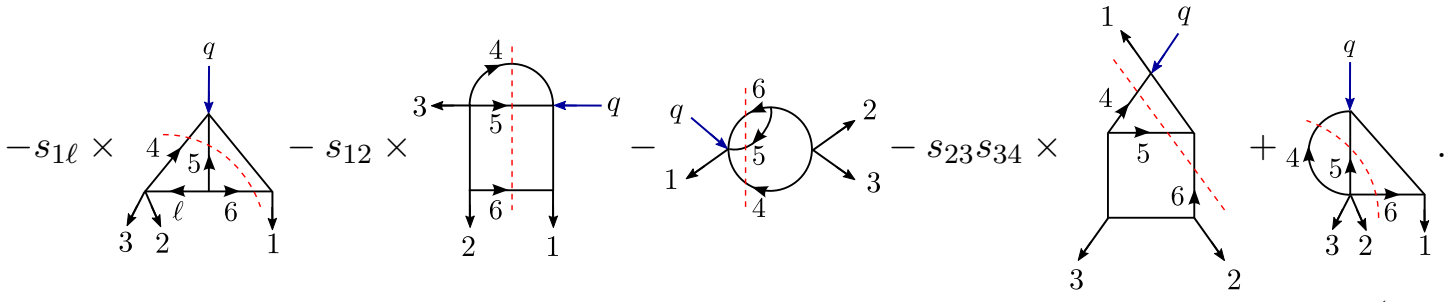

The complete contribution of the three-particle cut in the $s_{23}$-channel is then obtained by adding (3.28) and (3.30), and multiplying the result by two to take into account the second helicity configuration corresponding to $F^{\mathrm{MHV}} \times A^{\overline{\mathrm{MHV}}}$.

\subsection{Summary and integral reduction}

We now summarise the result of our calculation and present the result for the form factor of $\mathcal{O}_{B}=\operatorname{Tr}(X[Y, Z])$, which includes also the half-BPS component $\tilde{\mathcal{O}}_{\text {BPS }}=\operatorname{Tr}(X\{Y, Z\})$ computed in [26] and quoted in (3.1). The integral basis is shown in table 1. In terms of 


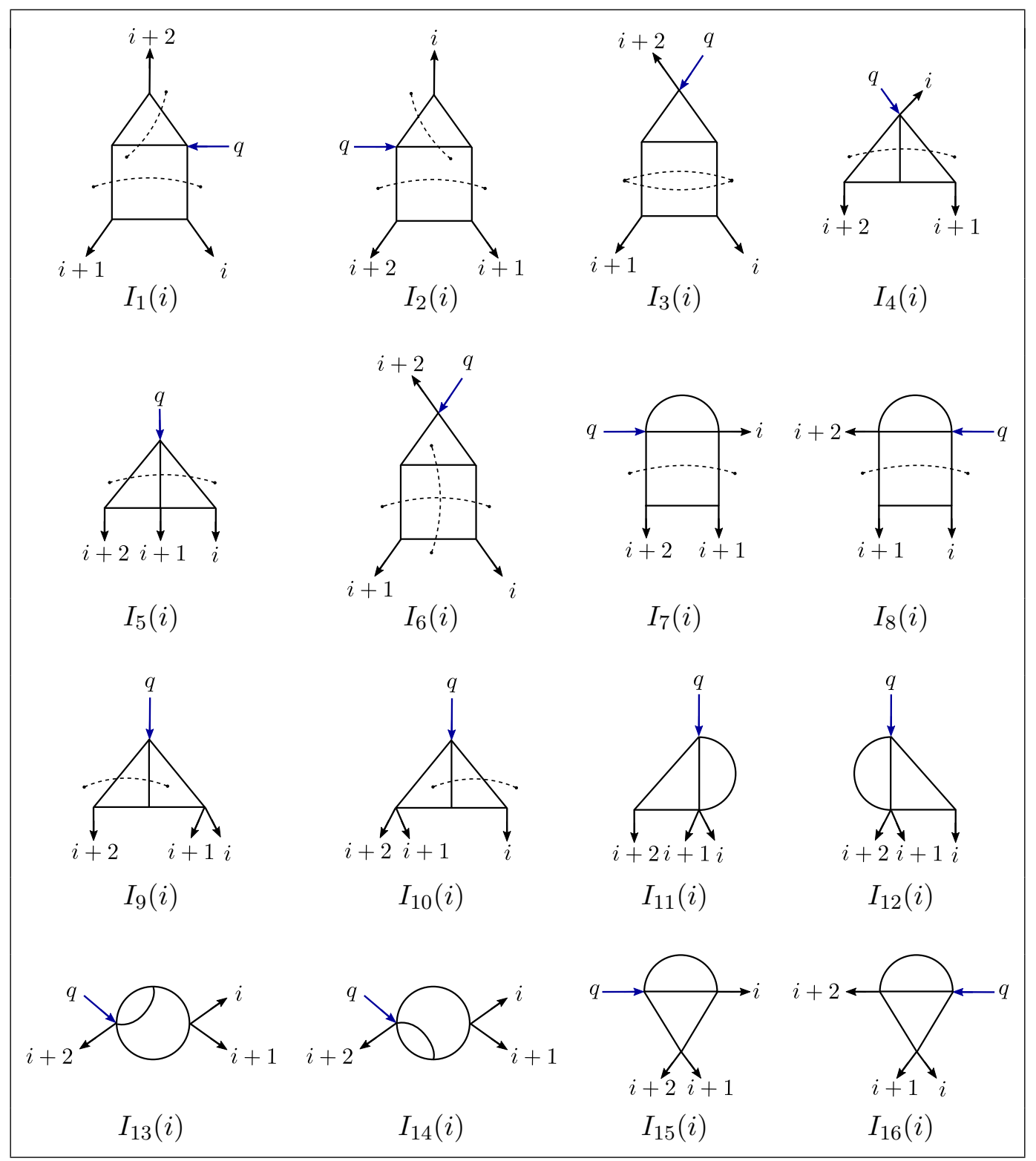

Table 1. Integral basis for the two-loop form factor $F_{\mathcal{O}_{B}}^{(2)}\left(1^{\phi^{12}}, 2^{\phi^{23}}, 3^{\phi^{31}} ; q\right)$. Note that the integrals $\left\{I_{1}(i), \ldots, I_{5}(i)\right\}$ correspond precisely to the BPS case, shown in eq. (3.25) of [26]. We use the same notation as in [26]: factors of $s_{i j} / s_{i j k}$ in the numerators are denoted by a dashed line intersecting two/three lines whose sum of momenta square to the corresponding kinematic invariant.

this basis, the two-loop minimal form factor of $\mathcal{O}_{B}$ is given by

$$
\begin{aligned}
F_{\mathcal{O}_{B}}^{(2)}\left(1^{\phi^{12}}, 2^{\phi^{23}}, 3^{\phi^{31}} ; q\right)= & -\sum_{i=1}^{4} I_{i}(1)+I_{5}(1)-2 \times\left[\sum_{i=6}^{10} I_{i}(1)-I_{11}(1)-I_{12}(1)\right. \\
& \left.+I_{13}(1)+I_{14}(1)\right]-4 \times\left[I_{15}(1)+I_{16}(1)\right] \\
& +\operatorname{cyclic}(1,2,3) .
\end{aligned}
$$


Some of the integrals appearing in (3.31) are master integrals and we can proceed to substitute their expressions from [41, 42]. The remaining ones will be reduced using a particular integration-by-parts algorithm implemented in the Mathematica package LiteRed [43, 44]. Using this package we find the following reductions:
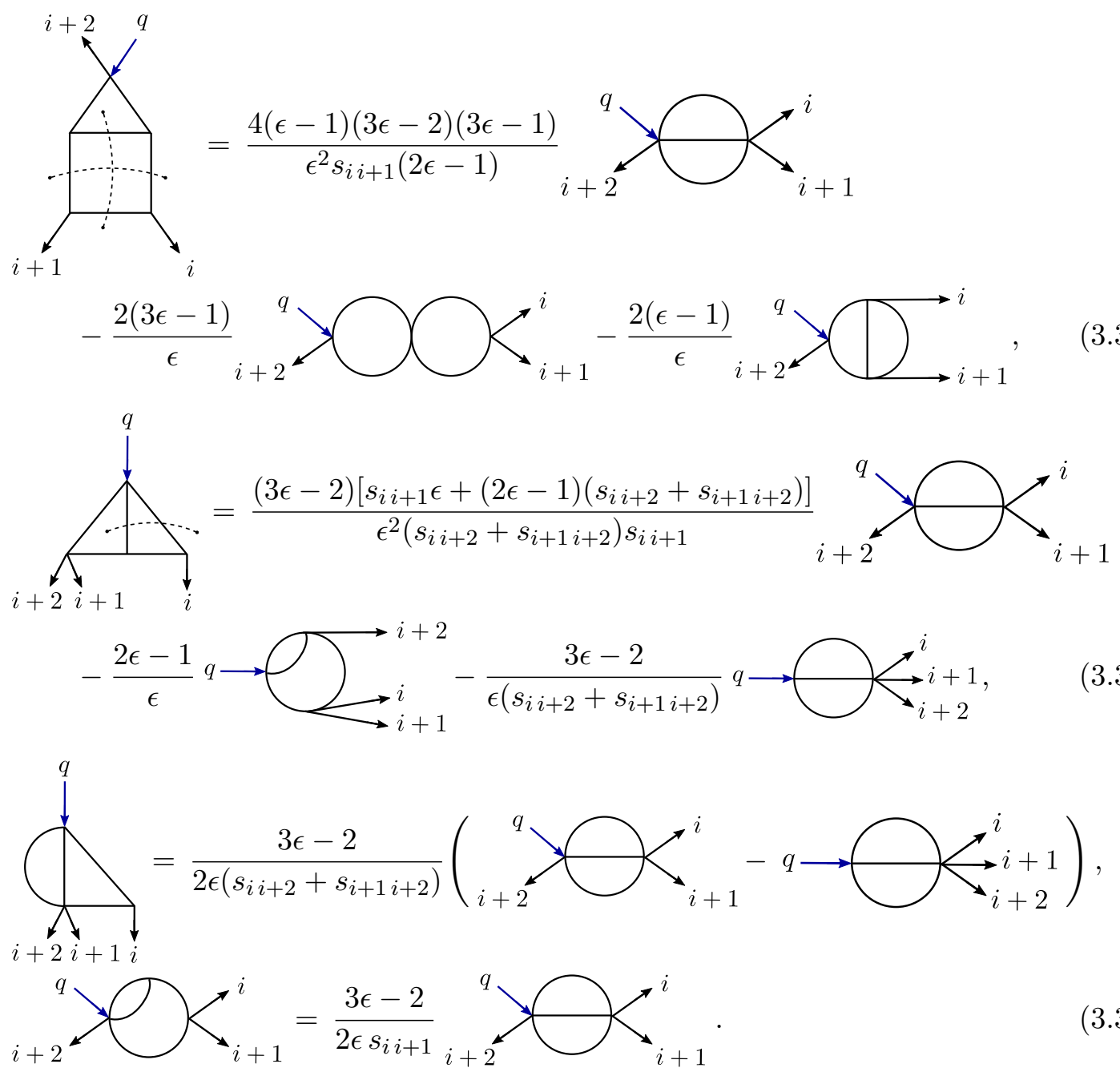

These reduced integrals, with expressions known from [41, 42], can then be plugged into (3.31) to give the final result of the two-loop form factor $F_{\mathcal{O}_{B}}^{(2)}\left(1^{\phi^{12}}, 2^{\phi^{23}}, 3^{\phi^{31}} ; q\right)$. We refrain from writing the full expression for this form factor at present due to its considerable length. Instead, we consider next a much simpler quantity obtained from a standard subtraction of the IR singularities - the remainder function.

\section{Two-loop remainder of $\langle\bar{X} \bar{Y} \bar{Z}|\operatorname{Tr} X[Y, Z]| 0\rangle$}

\subsection{Definition of the remainder}

Two-loop remainder functions for the form factor of a generic operator $\mathcal{O}$ were introduced in [6] similarly to the amplitude remainder function [45, 46],

$$
\mathcal{R}_{\mathcal{O}}^{(2)}:=F_{\mathcal{O}}^{(2)}(\epsilon)-\frac{1}{2}\left(F_{\mathcal{O}}^{(1)}(\epsilon)\right)^{2}-f^{(2)}(\epsilon) F_{\mathcal{O}}^{(1)}(2 \epsilon)-C^{(2)}+\mathcal{O}(\epsilon),
$$


where $f^{(2)}(\epsilon):=-2\left(\zeta_{2}+\epsilon \zeta_{3}+\epsilon^{2} \zeta_{4}\right)$ and $C^{(2)}=4 \zeta_{4}$. As in $[6,26]$, the function $f^{(2)}(\epsilon)$ is the same as for amplitudes $[45,46]$. Note that we have defined the remainder by taking out a power of

$$
\frac{g^{2} N e^{-\epsilon \gamma_{\mathrm{E}}}}{(4 \pi)^{2-\epsilon}}=a\left(4 \pi e^{-\gamma_{\mathrm{E}}}\right)^{\epsilon}
$$

per loop, where $a$ is our 't Hooft coupling, defined in (2.18). We also observe that in general we would define the remainder for the helicity-blind ratio $F_{\mathcal{O}}^{(2)} / F_{\mathcal{O}}^{(0)}$ as in [6] but in this particular case this is not necessary since the tree-level form factor is equal to one. An important aspect of this procedure $[6,45,46]$ is that it removes the universal IR divergences of the result. In the case of protected operators this gives a finite remainder while in the present case, where we consider a bare, unprotected operator, we are still left with UV divergences. In section 7 we will determine the appropriate renormalised operators and form factors that have a UV and IR finite remainder function. Here however we wish to take a first look at the IR-finite, but UV-divergent remainder function of the form factor $\langle\bar{X} \bar{Y} \bar{Z}|\operatorname{Tr}(X[Y, Z])| 0\rangle$.

Using the decomposition (2.6), the remainder function splits into a term formed completely by the form factor of $\tilde{\mathcal{O}}_{\text {BPS }}$ and a piece which contains mixed terms involving $\tilde{\mathcal{O}}_{\text {BPS }}$ and $\mathcal{O}_{\text {offset }}$, which we denote by $\mathcal{R}_{\text {non-BPS }}^{(2)}$ :

$$
\mathcal{R}_{\mathcal{O}_{B}}^{(2)}=\mathcal{R}_{\text {BPS }}^{(2)}+\mathcal{R}_{\text {non-BPS }}^{(2)},
$$

where

$$
\begin{aligned}
& \mathcal{R}_{\mathrm{BPS}}^{(2)}=F_{\tilde{\mathcal{O}}_{\mathrm{BPS}}}^{(2)}(\epsilon)-\frac{1}{2}\left(F_{\tilde{\mathcal{O}}_{\mathrm{BPS}}}^{(1)}(\epsilon)\right)^{2}-f^{(2)}(\epsilon) F_{\tilde{\mathcal{O}}_{\mathrm{BPS}}}^{(1)}(2 \epsilon)-C^{(2)}, \\
& \mathcal{R}_{\text {non-BPS }}^{(2)}=F_{\mathcal{O}_{\text {offset }}}^{(2)}(\epsilon)-F_{\mathcal{O}_{\text {offset }}}^{(1)}\left(\frac{1}{2} F_{\mathcal{O}_{\text {offset }}}^{(1)}+F_{\tilde{\mathcal{O}}_{\mathrm{BPS}}}^{(1)}\right)(\epsilon)-f^{(2)}(\epsilon) F_{\mathcal{O}_{\text {offset }}}^{(1)}(2 \epsilon) .
\end{aligned}
$$

The remainder of the half-BPS operator $\operatorname{Tr}\left(X^{3}\right)$ was computed in eq. (4.21) of [26] and is identical to the BPS remainder appearing here. It is given by a function of uniform transcendentality equal to four, written in terms of classical polylogarithms only. Explicitly, its expression is

$$
\begin{aligned}
\mathcal{R}_{\mathrm{BPS}}^{(2)}:= & \frac{3}{2} \operatorname{Li}_{4}(u)-\frac{3}{4} \operatorname{Li}_{4}\left(-\frac{u v}{w}\right)+\frac{3}{2} \log (w) \operatorname{Li}_{3}\left(-\frac{u}{v}\right)-\frac{1}{16} \log ^{2}(u) \log ^{2}(v) \\
& -\frac{\log ^{2}(u)}{32}\left[\log ^{2}(u)-4 \log (v) \log (w)\right]-\frac{\zeta_{2}}{8} \log (u)[5 \log (u)-2 \log (v)] \\
& -\frac{\zeta_{3}}{2} \log (u)-\frac{7}{16} \zeta_{4}+\operatorname{perms}(u, v, w)
\end{aligned}
$$

where

$$
u=\frac{s_{12}}{q^{2}}, \quad v=\frac{s_{23}}{q^{2}}, \quad w=\frac{s_{31}}{q^{2}}, \quad u+v+w=1 .
$$

The new part is the non-BPS remainder defined in (4.5). It is IR finite, but it still has UV divergences due to the fact that the operator inserted is not protected. Interestingly, it is 
given by a sum of functions of transcendentality ranging from three to zero, with no term with maximal transcendentality:

$$
\mathcal{R}_{\text {non-BPS }}^{(2)}=\frac{c}{\epsilon}+\sum_{i=0}^{3} \mathcal{R}_{\text {non-BPS;3-i }}^{(2)},
$$

where the subscript $m$ in $\mathcal{R}_{\text {non-BPS; } m}^{(2)}$ denotes the degree of transcendentality of the corresponding term. For the coefficient of the UV pole we find

$$
c=18-\pi^{2} .
$$

The expression arising from replacing the integral functions appearing in the two-loop form factor with the explicit results of $[41,42]$ can be considerably simplified using the concept of the symbol of a transcendental function [47], while beyond-the-symbol terms can be fixed numerically and/or analytically. At transcendentality three, we are guaranteed that the whole result can be written in terms of classical polylogarithms only, and hence this procedure is very simple to carry out. We find that the symbol of $\mathcal{R}_{\text {non-BPS;3 }}^{(2)}$ is

$\mathcal{S}_{3}^{(2)}(u, v, w)=-2\left[u \otimes(1-u) \otimes \frac{u}{1-u}+u \otimes u \otimes \frac{v}{1-u}+u \otimes v \otimes \frac{u v}{w^{2}}\right]+\operatorname{perms}(u, v, w)$,

while for the integrated expression (including beyond-the-symbol terms) we get

$$
\begin{aligned}
\mathcal{R}_{\text {non-BPS; } 3}^{(2)}= & 2\left[\operatorname{Li}_{3}(u)+\operatorname{Li}_{3}(1-u)\right]-\frac{1}{2} \log ^{2}(u) \log \frac{v w}{(1-u)^{2}}+\frac{2}{3} \log (u) \log (v) \log (w) \\
& +\frac{2}{3} \zeta_{3}+2 \zeta_{2} \log \left(-q^{2}\right)+\operatorname{perms}(u, v, w)
\end{aligned}
$$

The transcendentality-two part of the remainder can also be simplified slightly. A short calculation leads to the expression

$$
\mathcal{R}_{\text {non-BPS;2 }}^{(2)}=-12\left[\operatorname{Li}_{2}(1-u)+\operatorname{Li}_{2}(1-v)+\operatorname{Li}_{2}(1-w)\right]-2 \log ^{2}(u v w)+36 \zeta_{2} .
$$

Finally, for the transcendentality-one and zero terms we have

$$
\begin{aligned}
& \mathcal{R}_{\text {non-BPS; } 1}^{(2)}=-12 \log (u v w)-36 \log \left(-q^{2}\right) \\
& \mathcal{R}_{\text {non-BPS;0 }}^{(2)}=126 .
\end{aligned}
$$

Before concluding this section we would like to make two observations on the results we have derived here.

1. First, we observe that the $-\pi^{2}$ term in (4.9) comes from the last term on the righthand side of (4.5). It amounts to introducing a spurious UV divergence in the remainder arising from the bubbles contained in the term $F_{\mathcal{O}_{\text {offset }}}^{(1)}(2 \epsilon)$. For the sake of extracting the correct UV divergences and studying the mixing, this term must be omitted, see section 7 for this discussion. 
2. We stress the usefulness of the decomposition (2.6) and (4.3), which has the great advantage of separating out completely the terms of maximal transcendentality from the rest. This is in line with the findings of [30], where it was observed in the $\mathrm{SU}(2)$ sector that a the finite remainder densities introduced there, and corresponding to different "shuffling" for the $R$-symmetry fields flavours, have a highest degree of transcendentality equal to $4-s$ with $s$ being the shuffling in that remainder density. In the present case, in a different sector, the operator $\operatorname{Tr}(X Z Y)$ is associated with an external state $\langle\bar{X} \bar{Y} \bar{Z}|$, which corresponds to $s=1$. Indeed we find that the corresponding remainder is composed of terms with transcendentality ranging from three to zero.

\subsection{A connection to the remainder densities in the $\mathrm{SU}(2)$ sector}

We now establish a connection between the (UV-finite part of the) non-BPS remainder $\mathcal{R}_{\text {non-BPS }}^{(2)}$ and the remainder densities which have appeared in [30] in connection with the calculation of the dilatation operator in the $\mathrm{SU}(2)$ sector. This is a closed subsector of $\mathrm{SU}(2 \mid 3)$ and operators are built out of the complex scalars $X$ and $Y$ defined earlier in (1.5). Two observations are in order here. Firstly we note that the remainder densities studied in [30] correspond to operators which are products of fields without the trace. Secondly, the operator we are considering is part of the larger $\mathrm{SU}(2 \mid 3)$ sector, hence we should not expect to find similarities with results obtained in smaller sectors. In particular, in the $\mathrm{SU}(2 \mid 3)$ sector the spin chain becomes dynamic i.e. the number of spin sites can fluctuate due to length-changing interactions, something which cannot occur in the $\mathrm{SU}(2)$ sector. We will see in section 7 that this is important for the renormalisation of the form factor of $\mathcal{O}_{B}$.

It was found in [30] that there are only three independent finite remainder densities, denoted in that paper as $\left(R_{i}^{(2)}\right)_{X X X}^{X X X},\left(R_{i}^{(2)}\right)_{X X Y}^{X Y X}$, and $\left(R_{i}^{(2)}\right)_{X X Y}^{Y X X}$. The first density, $\left(R_{i}^{(2)}\right)_{X X X X}^{X X X}$, has uniform transcendentality equal to four and is identical to the half-BPS remainder computed in [26]. $\left(R_{i}^{(2)}\right)_{X X Y}^{X Y X}$ contains terms of transcendentality ranging from three to zero, while $\left(R_{i}^{(2)}\right)_{X X Y}^{Y X X}$ contains terms of transcendentality two, one and zero. The index $i$ denotes the spin chain site, and the remainder densities depend on the three variables

$$
u_{i}=\frac{s_{i i+1}}{s_{i i+1 i+2}}, \quad v_{i}=\frac{s_{i+1 i+2}}{s_{i i+1 i+2}}, \quad w_{i}=\frac{s_{i i+2}}{s_{i i+1 i+2}},
$$

as well as on $s_{i i+1}, s_{i+2 i+2}, s_{i i+2}$ and $s_{i i+1 i+2}$ separately.

We have observed an interesting connection between these remainder densities and our non-BPS remainder, namely

$$
\begin{aligned}
& \frac{1}{2} \mathcal{R}_{\text {non-BPS; } 3}^{(2)}=-\left.\sum_{S_{3}}\left(R_{i}^{(2)}\right)_{X X Y}^{X Y X}\right|_{3}+6 \zeta_{3}, \\
& \frac{1}{2} \mathcal{R}_{\text {non-BPS; } 2}^{(2)}=-\left.\sum_{S_{3}}\left[\left(R_{i}^{(2)}\right)_{X X Y}^{X Y X}-\left(R_{i}^{(2)}\right)_{X X Y}^{Y X X}\right]\right|_{2}+5 \pi^{2}, \\
& \frac{1}{2} \mathcal{R}_{\text {non-BPS } ; 1}^{(2)}=-\left.\sum_{S_{3}}\left[\left(R_{i}^{(2)}\right)_{X X Y}^{X Y X}-\left(R_{i}^{(2)}\right)_{X X X}^{Y X X}\right]\right|_{1}, \\
& \frac{1}{2} \mathcal{R}_{\text {non-BPS;0}}^{(2)}=-\left.\sum_{S_{3}}\left[\left(R_{i}^{(2)}\right)_{X X Y}^{X Y X}-\left(R_{i}^{(2)}\right)_{X X Y}^{Y X X}\right]\right|_{0},
\end{aligned}
$$




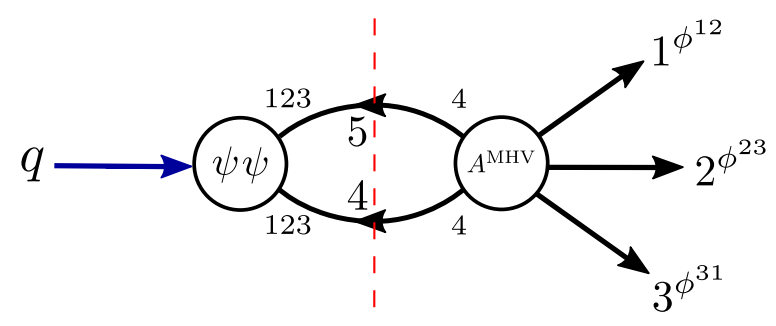

Figure 11. Two-particle cut of the non-minimal form factor $F_{\mathcal{O}_{F}}^{(1)}$ with external state $\langle\bar{X} \bar{Y} \bar{Z}|$.

where $\left.f\right|_{m}$ denotes the transcendentality- $m$ part of the function $f$, the remainder densities are evaluated with the replacements $\left(u_{i}, v_{i}, w_{i}\right) \rightarrow(u, v, w)$, and $S_{3}$ denotes permutations of $(u, v, w)$. It would be very interesting to explain this almost perfect coincidence of these a priori unrelated quantities.

\section{One-loop non-minimal form factor $\left\langle\bar{X} \bar{Y} \bar{Z}\left|\frac{1}{2} \operatorname{Tr} \psi^{\alpha} \psi_{\alpha}\right| 0\right\rangle$}

In this section we compute one of the off-diagonal entries of the matrix of form factors $(1.10)$, namely $F_{\mathcal{O}_{F}}^{(1)}\left(1^{\phi^{12}}, 2^{\phi^{23}}, 3^{\phi^{31}} ; q\right)$, where $\mathcal{O}_{F}=(1 / 2) \operatorname{Tr}\left(\psi^{\alpha} \psi_{\alpha}\right)$. Note that $\mathcal{O}_{F}$ is defined in a way that its minimal tree-level form factor $\left\langle\bar{\psi}^{123}(1) \bar{\psi}^{123}(2)\left|\mathcal{O}_{F}(0)\right| 0\right\rangle$ is equal to $\langle 21\rangle$.

In order to do so we construct the one-loop integrand by considering two-particle cuts in the $q^{2}$ and $s_{23}$ channels. We will find that the result is IR finite as it should be since this form factor does not exist at tree level. However, UV divergences are expected reflecting the mixing between $\mathcal{O}_{B}$ and $\mathcal{O}_{F}$. This will be studied in detail in section 7 .

\subsection{Two-particle cut in the $q^{2}$-channel}

We start by computing the $q^{2}$-channel of the form factor $F_{\mathcal{O}_{F}}^{(1)}\left(1^{\phi^{12}}, 2^{\phi^{23}}, 3^{\phi^{31}} ; q\right)$. This is shown in figure 11 and is given by

$$
\begin{aligned}
&\left.F_{\mathcal{O}_{F}}^{(1)}\left(1^{\phi^{12}}, 2^{\phi^{23}}, 3^{\phi^{31}} ; q\right)\right|_{2, q^{2}}=i^{2} F_{\mathcal{O}_{F}}^{(0)}\left(-5^{\bar{\psi}^{123}},-4^{\bar{\psi}^{123}} ; q\right) \times A\left(1^{\phi^{12}}, 2^{\phi^{23}}, 3^{\phi^{31}}, 4^{\psi^{4}}, 5^{\psi^{4}}\right) \\
&=-i\langle 45\rangle \times \frac{\langle 13\rangle}{\langle 34\rangle\langle 51\rangle}=-\frac{i}{2}\left(\frac{s_{34} s_{15}+s_{45} s_{13}-s_{14} s_{35}}{s_{34} s_{15}}\right) .
\end{aligned}
$$

The corresponding topology is the box shown in figure 12 .

We now rewrite the numerators in (5.1) using

$$
s_{45}=s_{123}, \quad s_{14}=-\left(s_{12}+s_{13}+s_{15}\right), \quad s_{35}=-\left(s_{31}+s_{32}+s_{34}\right),
$$

which follow from momentum conservation $\sum_{i=1}^{5} p_{i}=0$ and the cut conditions 


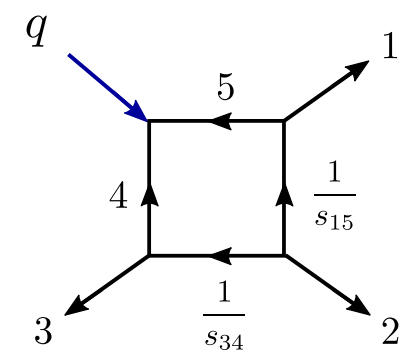

Figure 12. The integral topology that appears in the $q^{2}$-channel two-particle cut. For future convenience we indicate explicitly the uncut propagators.

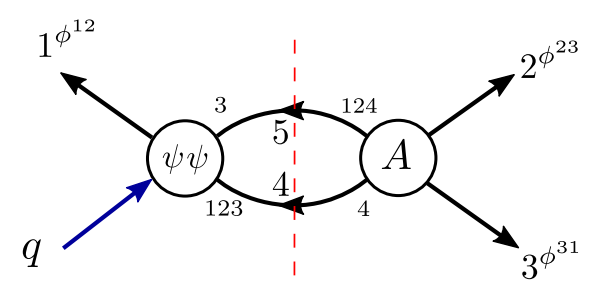

(i)

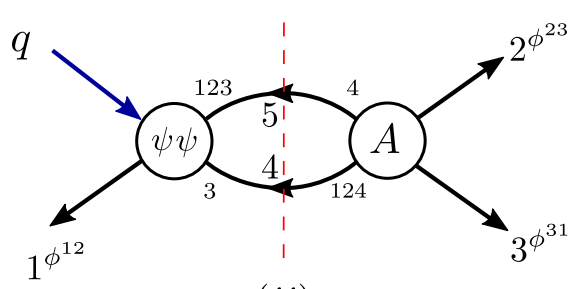

$(i i)$

Figure 13. Two diagrams entering the two-particle cut in the $s_{23}$-channel.

$p_{4}^{2}=p_{5}^{2}=0$. Doing so $(5.1)$ becomes

$$
\begin{aligned}
& \left.F_{\mathcal{O}_{F}}^{(1)}\left(1^{\phi^{12}}, 2^{\phi^{23}}, 3^{\phi^{31}} ; q\right)\right|_{2, q^{2}}=\frac{i}{2}\left(\frac{s_{12} s_{23}}{s_{34} s_{15}}+\frac{s_{13}+s_{23}}{s_{34}}+\frac{s_{12}+s_{13}}{s_{15}}\right) \\
& =\frac{i}{2}\left[s_{12} s_{23} \times s_{2}^{q}+\left(s_{13}+s_{23}\right) \times s_{2}^{q}+s_{12}+s_{13}\right) \times \underbrace{q}_{2}] .
\end{aligned}
$$

Note that in this cut no UV-divergent integrals have appeared and we have to add two additional contributions from cyclic permutations of the external particles.

\subsection{Two-particle cut in the $s_{23}$-channel}

We now compute the two-particle cut of $F_{\mathcal{O}_{F}}^{(1)}\left(1^{\phi^{12}}, 2^{\phi^{23}}, 3^{\phi^{31}} ; q\right)$ in the $s_{23}$-channel. There are two possible diagrams to consider, shown in figure 13 .

These two diagrams give rise to the master topologies shown in figure 14, with corresponding numerators determined by the cuts.

In the cuts we need the tree-level non-minimal form factors $F_{\mathcal{O}_{F}}^{(0)}\left(1^{\phi^{12}}, 2^{\psi^{3}}, 3^{\bar{\psi}^{123}} ; q\right)$ and $F_{\mathcal{O}_{F}}^{(0)}\left(1^{\phi^{12}}, 2^{\psi^{123}}, 3^{\bar{\psi}^{3}} ; q\right)$. The first of them has only one possible factorisation diagram corresponding to a fermion splitting into an anti-fermion and a scalar, as shown in figure 15.

From this factorisation diagram we can infer the expression for the tree-level form factor, which is given by

$$
F_{\mathcal{O}_{F}}^{(0)}\left(1^{\phi^{12}}, 2^{\psi^{3}}, 3^{\bar{\psi}^{123}} ; q\right)=F_{\mathcal{O}_{F}}^{(0)}\left(-4^{\bar{\psi}^{123}}, 3^{\bar{\psi}^{123}} ; q\right) \times \frac{i}{s_{12}} \times A^{\overline{\mathrm{MHV}}}\left(1^{\phi^{12}}, 2^{\psi^{3}}, 4^{\psi^{4}}\right) .
$$




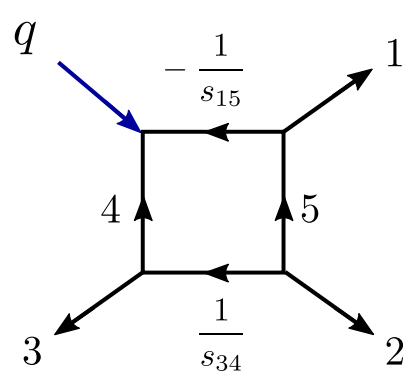

(i)

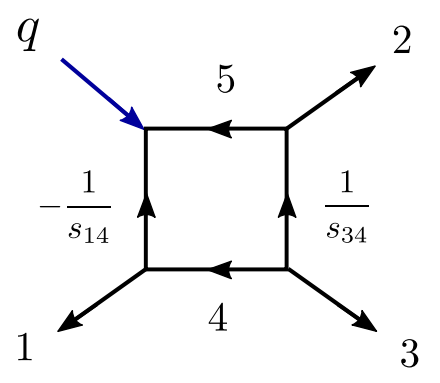

(ii)

Figure 14. Master topologies generated by the two diagrams of figure 13, respectively. The uncut propagators are explicitly shown in order to bookkeep their sign reflecting the momentum flow. For the coefficient of the box integral, only the diagram on the left can be compared with the box detected in the $q^{2}$-cut of figure 11 due to the ordering of external legs.

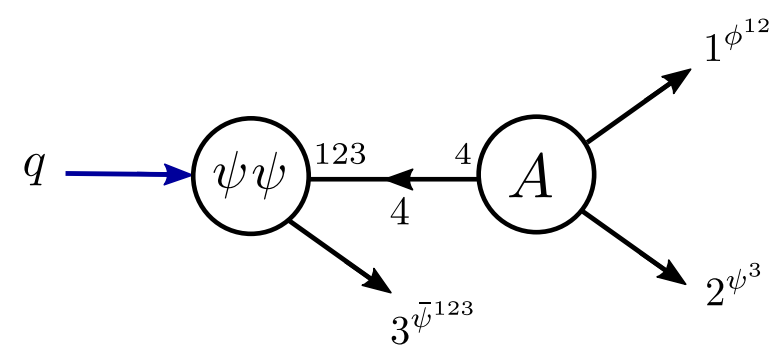

Figure 15. A factorisation diagram of the non-minimal form factor $F_{\mathcal{O}_{F}}^{(0)}\left(1^{\phi^{12}}, 2^{\psi^{3}}, 3^{\bar{\psi}^{123}} ; q\right)$ featuring in the two-particle cut of $F_{\mathcal{O}_{F}}^{(1)}\left(1^{\phi^{12}}, 2^{\phi^{23}}, 3^{\phi^{31}} ; q\right)$ in the $s_{23}$-channel.

The anti-MHV amplitude can be easily determined using parity,

$$
A^{\overline{\mathrm{MHV}}}\left(1^{\phi^{12}}, 2^{\psi^{3}}, 4^{\psi^{4}}\right)=-\left[A^{\mathrm{MHV}}\left(1^{\phi^{34}}, 2^{\bar{\psi}^{124}}, 4^{\bar{\psi}^{123}}\right)\right]^{*}=i[24] .
$$

Using $p_{4}=-\left(p_{1}+p_{2}\right)$ we obtain the result

$$
F_{\mathcal{O}_{F}}^{(0)}\left(1^{\phi^{12}}, 2^{\psi^{3}}, 3^{\bar{\psi}^{123}} ; q\right)=\frac{[21]\langle 13\rangle}{s_{12}}
$$

We now compute the two diagrams of figure 13 separately.

Diagram $(\boldsymbol{i})$. This diagram is given by

$$
\begin{aligned}
\left.F_{\mathcal{O}_{F}}^{(1)}\left(1^{\phi^{12}}, 2^{\phi^{23}}, 3^{\phi^{31}} ; q\right)\right|_{2, s_{23},(i)} & =-i^{2} F_{\mathcal{O}_{F}}^{(0)}\left(1^{\phi^{12}},-5^{\psi^{3}},-4^{\bar{\psi}^{123}} ; q\right) \times A^{\mathrm{MHV}}\left(2^{\phi^{23}}, 3^{\phi^{31}}, 4^{\psi^{4}}, 5^{\bar{\psi}^{124}}\right) \\
& =-\frac{i}{2}\left(\frac{s_{14} s_{35}+s_{34} s_{15}-s_{13} s_{45}}{s_{15} s_{34}}\right)
\end{aligned}
$$

Using $p_{2}+p_{3}+p_{4}+p_{5}=0$ and $p_{4}^{2}=p_{5}^{2}=0$ on the cut, we can substitute

$$
s_{45}=s_{23}, \quad s_{35}=-\left(s_{34}+s_{32}\right), \quad s_{14}=-\left(s_{12}+s_{13}+s_{15}\right)
$$


thus (5.7) becomes

$$
\begin{aligned}
& \left.F_{\mathcal{O}_{F}}^{(1)}\left(1^{\phi^{12}}, 2^{\phi^{23}}, 3^{\phi^{31}} ; q\right)\right|_{2, s_{23},(i)}=-\frac{i}{2}\left[2+\frac{s_{12}+s_{13}}{s_{15}}+\frac{s_{23}}{s_{34}}+\frac{s_{12} s_{23}}{s_{15} s_{34}}\right] \\
& =\frac{i}{2}[-2 \times{ }_{1}^{q}+\left(s_{12}+s_{13}\right) \times \underbrace{2}_{32}-s_{23} \times \underbrace{q}_{3}+s_{12}^{2} s_{23} \times \underbrace{1}_{2}] \text {. }
\end{aligned}
$$

Note that when the cut-integrals are uplifted to full Feynman integrals $1 / s_{15}$, has to be replaced by $-1 /\left(p_{1}-p_{5}\right)^{2}$ due to the momentum flow, according to figure $14(i)$.

Diagram (ii). For diagram $(i i)$ we need the form factor

$$
F_{\mathcal{O}_{F}}^{(0)}\left(1^{\phi^{12}},-5^{\bar{\psi}^{123}},-4^{\psi^{3}} ; q\right)=F_{\mathcal{O}_{F}}^{(0)}\left(1^{\phi^{12}},-4^{\psi^{3}},-5^{\bar{\psi}^{123}} ; q\right)=\frac{[41]\langle 15\rangle}{s_{14}} .
$$

Its expression is given by

$$
\begin{gathered}
\left.F_{\mathcal{O}_{F}}^{(1)}\left(1^{\phi^{12}}, 2^{\phi^{23}}, 3^{\phi^{31}} ; q\right)\right|_{2, s_{23},(i i)}=-i^{2} F_{\mathcal{O}_{F}}^{(0)}\left(1^{\phi^{12}},-5^{\bar{\psi}^{123}},-4^{\psi^{3}} ; q\right) \times A^{\mathrm{MHV}}\left(2^{\phi^{23}}, 3^{\phi^{31}}, 4^{\bar{\psi}^{124}}, 5^{\psi^{4}}\right) \\
=i \frac{[41]\langle 15\rangle}{s_{14}} \times\left(\frac{\langle 24\rangle}{\langle 52\rangle}\right)=-i \frac{\operatorname{Tr}_{+}(1524)}{s_{14} s_{25}}=i \frac{\operatorname{Tr}_{+}(1534)}{s_{14} s_{34}}
\end{gathered}
$$

where we used momentum conservation in the last step. Expanding the trace and using a set of replacements similar to (5.8),

$$
s_{45}=s_{23}, \quad s_{35}=-\left(s_{34}+s_{32}\right), \quad s_{15}=-\left(s_{12}+s_{13}+s_{14}\right),
$$

we arrive at the result

$$
\left.F_{\mathcal{O}_{F}}^{(1)}\left(1^{\phi^{12}}, 2^{\phi^{23}}, 3^{\phi^{31}} ; q\right)\right|_{2, s_{23},(i i)}=-\frac{i}{2}\left[2+\frac{s_{12}+s_{13}}{s_{14}}+\frac{s_{23}}{s_{34}}+\frac{s_{13} s_{23}}{s_{14} s_{34}}\right]
$$

which is identical to (5.9) apart from the box. Note that in the sum over cyclic permutations of these two cuts three different one-mass boxes appear, each with their two possible two-particle cuts. The cuts of the same boxes in the $q^{2}$-channel are already accounted for in (5.3).

Diagram (i) + Diagram (ii). Combining the results (5.9) and (5.13) and noting that the coefficients of the integrals are consistent with those obtained from the $q^{2}$-channel cut 
in (5.3), we find

$$
\left.F_{\mathcal{O}_{F}}^{(1)}\left(1^{\phi^{12}}, 2^{\phi^{23}}, 3^{\phi^{31}} ; q\right)\right|_{2, s_{23}}=\left.F_{\mathcal{O}_{F}}^{(1)}\left(1^{\phi^{12}}, 2^{\phi^{23}}, 3^{\phi^{31}} ; q\right)\right|_{2, s_{23},(i)}+\left.F_{\mathcal{O}_{F}}^{(1)}\left(1^{\phi^{12}}, 2^{\phi^{23}}, 3^{\phi^{31}} ; q\right)\right|_{2, s_{23},(i i)}
$$

Note that the coefficient of the box integral with $q$ inserted between $p_{1}$ and $p_{3}$ matches that obtained in the $q^{2}$-channel (5.3), namely $(i / 2)\left(s_{12} s_{23}\right)$. Moreover, the second box appearing in (5.14) is detected in the $q^{2}$-cut with cyclically shifted external momenta: $1 \rightarrow 2 \rightarrow 3 \rightarrow 1$.

\subsection{Final result}

Performing the cyclic sum we get the final result for the one-loop form factor:

$$
F_{\mathcal{O}_{F}}^{(1)}\left(1^{\phi^{12}}, 2^{\phi^{23}}, 3^{\phi^{31}} ; q\right)=\frac{i}{2}[-4 \times \overbrace{3}^{q}+2 s_{23} \times \overbrace{2}^{2}+s_{12} s_{23} \times s_{2}^{1}+\operatorname{cyclic}(1,2,3)] .
$$

Expanding the result to $\mathcal{O}(\epsilon)$ we get

$$
\begin{aligned}
F_{\mathcal{O}_{F}}^{(1)}\left(1^{\phi^{12}}, 2^{\phi^{23}}, 3^{\phi^{31}} ; q\right)= & \frac{6}{\epsilon}+12+\frac{\pi^{2}}{2}-\left[2 \log \left(-s_{12}\right)-\frac{1}{2} \log ^{2} \frac{s_{12}}{s_{23}}\right. \\
& \left.-2 \operatorname{Li}_{2}\left(1-\frac{q^{2}}{s_{12}}\right)+\operatorname{cyclic}(1,2,3)\right]+\mathcal{O}(\epsilon)
\end{aligned}
$$

Importantly the infrared $1 / \epsilon^{2}$ poles have cancelled in the final result, which is expected since the corresponding tree-level form factor does not exist. We can also rewrite the result using the variables $u, v$ ad $w$ introduced in (4.7), getting ${ }^{13}$

$$
F_{\mathcal{O}_{F}}^{(1)}\left(1^{\phi^{12}}, 2^{\phi^{23}} 3^{\phi^{31}} ; q\right)=2 \frac{\left(-s_{12}\right)^{-\epsilon}}{\epsilon(1-2 \epsilon)}-\left[2 \operatorname{Li}_{2}(1-u)+\log u \log v\right]+\zeta_{2}+\operatorname{cyclic}(1,2,3) .
$$

\footnotetext{
${ }^{13}$ Note that under renormalisation this quantity will combine with (4.12).
} 


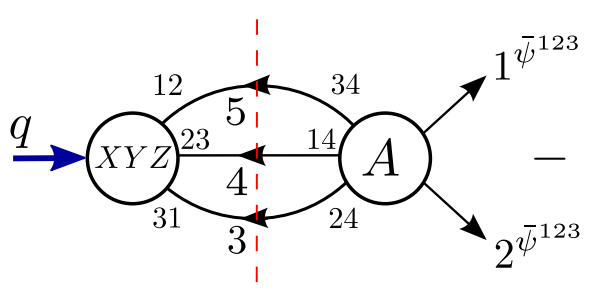

(i)

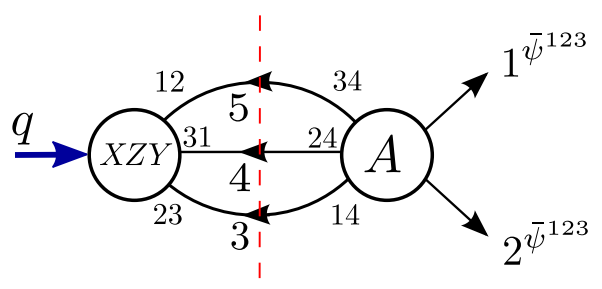

(ii)

Figure 16. Triple cut of the two-loop sub-minimal form factor $\left\langle\bar{\psi} \bar{\psi}\left|\mathcal{O}_{B}\right| 0\right\rangle$. The second set of identical diagrams, but with external legs 1 and 2 swapped has to be added, corresponding to the fact that it leads to the same colour-ordering.

\section{Two-loop sub-minimal form factor $\langle\bar{\psi} \bar{\psi}|\operatorname{Tr} X[Y, Z]| 0\rangle$}

Here we consider the second off-diagonal form factor in (1.10), namely the sub-minimal form factor $\left\langle\bar{\psi} \bar{\psi}\left|\mathcal{O}_{B}\right| 0\right\rangle$ with $\mathcal{O}_{B}=\operatorname{Tr}(X[Y, Z])$ and $\langle\bar{\psi} \bar{\psi}|$ being a shorthand notation for $\left\langle 1^{123} 2^{\bar{\psi}^{123}}\right|$. As it is clear from figure 16, this object exists only at two loops or more, hence we only need to consider the two three-particle cuts presented here.

For the first diagram, the relevant amplitude (and hence the integrand, since the treelevel form factor is just 1 ) is

$$
(i): A\left(1^{\bar{\psi}^{123}}, 2^{\bar{\psi}^{123}}, 3^{\phi^{24}}, 4^{\phi^{14}}, 5^{\phi^{34}}\right)=-i \frac{[53]}{[23][51]} .
$$

For the second diagram, the relevant amplitude is

$$
(i i): A\left(1^{\bar{\psi}^{123}}, 2^{\bar{\psi}^{123}}, 3^{\phi^{14}}, 4^{\phi^{24}}, 5^{\phi^{34}}\right)=i \frac{[53]}{[23][51]},
$$

which differs from $(i)$ only by a sign. Taking into account the relative minus sign between the two diagrams coming from the commutator and converting to momentum invariants we get

$$
(i)-(i i): \frac{1}{[12]} \cdot \frac{s_{35} s_{12}-s_{25} s_{13}+s_{15} s_{23}}{s_{23} s_{15}},
$$

where we have taken into account the factor of $i^{3}$ coming from the cut propagators. We note that for the half-BPS case of $\tilde{\mathcal{O}}_{\mathrm{BPS}}=\operatorname{Tr}(X\{Y, Z\})$ the two contributions would cancel out exactly, which is consistent with the fact that the operator is protected.

The cut integrand corresponding to the expression in (6.3) is given by

$$
\left.F_{\mathcal{O}_{B}}^{(2)}\left(1^{\bar{\psi}^{123}}, 2^{\bar{\psi}^{123}} ; q\right)\right|_{3, q^{2}}=\frac{1}{[12]}\left(s_{35} s_{12}-s_{25} s_{13}+s_{15} s_{23}\right){ }_{1}
$$


Lifting the cut momenta off-shell and performing the integral reductions using the LiteRed package gives an $\epsilon$-dependent prefactor times a "sunset" integral,

$$
F_{\mathcal{O}_{B}}^{(2)}\left(1^{\bar{\psi}^{123}}, 2^{\bar{\psi}^{123}} ; q\right)=\frac{1}{[12]} \frac{2(3 \epsilon-2)}{2 \epsilon-1} \times q \longrightarrow
$$

Note that any ambiguity associated with factors of $p_{i}^{2}, i=4,5,6$ in the numerator of (6.4) would lead to a (vanishing) scaleless integral.

Finally, we proceed to substitute the expression for the sunset integral, which can be found in [41]. We also perform a summation over the cyclic permutations of the internal legs and note that having done so, the value of the five-point amplitude entering the cut does not change and so the result picks up an overall factor of three. Finally, a further factor of two is included corresponding to the two possible orderings of the external legs.

We proceed by expanding the results in powers of $\epsilon$ up to $\mathcal{O}(\epsilon)$ and get

$$
\begin{aligned}
F_{\mathcal{O}_{B}}^{(2)}\left(1^{\bar{\psi}^{123}}, 2^{\bar{\psi}^{123}} ; q\right) & =\frac{6}{[12]} \frac{\epsilon^{2}}{(1-2 \epsilon)^{2}} \frac{\Gamma(1+2 \epsilon) \Gamma(-\epsilon)^{3}}{\Gamma(2-3 \epsilon)}\left(e^{\gamma_{\mathrm{E}} \epsilon}\right)^{2}\left(-q^{2}\right)^{1-2 \epsilon} \\
& =-6\langle 12\rangle\left[\frac{1}{\epsilon}+7-2 \log \left(-q^{2}\right)\right]+\mathcal{O}(\epsilon)
\end{aligned}
$$

Note that this sub-minimal two-loop form factor has no lower-loop counterparts and, therefore, it has only a $1 / \epsilon$ UV divergence and no IR divergences.

\section{Two-loop dilatation operator in the $\mathrm{SU}(2 \mid 3)$ sector}

In this section we resolve the mixing between the two operators $\mathcal{O}_{B}=\operatorname{Tr}(X[Y, Z])$ and $\mathcal{O}_{F}=(1 / 2) \operatorname{Tr}\left(\psi^{\alpha} \psi_{\alpha}\right)$ at two loops. Recall that all other dimension-three operators in the $\mathrm{SU}(2 \mid 3)$ sector such as $\operatorname{Tr}\left(X^{3}\right), \operatorname{Tr}\left(X^{2} Y\right)$ and $\tilde{\mathcal{O}}_{\mathrm{BPS}}=\operatorname{Tr}(X\{Y, Z\})$ are half-BPS and do not mix. Doing so we will reproduce the two-loop dilatation operator for these operators in the $\mathrm{SU}(2 \mid 3)$ sector originally derived in [28].

We introduce the renormalised operators

$$
\left(\begin{array}{c}
\mathcal{O}_{F}^{\text {ren }} \\
\mathcal{O}_{B}^{\text {ren }}
\end{array}\right)=\left(\begin{array}{ll}
\mathcal{Z}_{F}^{F} & \mathcal{Z}_{F}{ }^{B} \\
\mathcal{Z}_{B}^{F} & \mathcal{Z}_{B}{ }^{B}
\end{array}\right)\left(\begin{array}{l}
\mathcal{O}_{F} \\
\mathcal{O}_{B}
\end{array}\right),
$$

where $\mathcal{O}_{F}$ and $\mathcal{O}_{B}$ are the bare operators that we used to compute form factors in earlier sections. The matrix of renormalisation constants $\mathcal{Z}$, also called mixing matrix, is determined by requiring the UV-finiteness of the form factors of the renormalised operators $\mathcal{O}_{F}^{\text {ren }}$ and $\mathcal{O}_{B}^{\text {ren }}$ with the external states $\langle\bar{X} \bar{Y} \bar{Z}|$ and $\langle\bar{\psi} \bar{\psi}|$. The quantum correction to the dilatation operator $\mathfrak{D}$, denoted by $\delta \mathfrak{D}$, is related to the mixing matrix $\mathcal{Z}$ as

$$
\delta \mathfrak{D}=-\mu_{R} \frac{\partial}{\partial \mu_{R}} \log \mathcal{Z}
$$


where $\mu_{R}$ is the renormalisation scale. The relevant form factors from which we extract the renormalisation constants are written below, and we also indicate the schematic form of their perturbative expansions, as reflected by perturbative calculations:

$$
\begin{aligned}
\left.\left\langle\bar{\psi} \bar{\psi}\left|\mathcal{O}_{F}\right| 0\right\rangle\right|_{\mathrm{UV}} & :=\langle 21\rangle\left[f^{(1)} a\left(\mu_{R}\right)+f^{(2)} a^{2}\left(\mu_{R}\right)+\cdots\right] \\
\left.\left\langle\bar{X} \bar{Y} \bar{Z}\left|\mathcal{O}_{F}\right| 0\right\rangle\right|_{\mathrm{UV}} & :=a\left(\mu_{R}\right)[g \cdot h]+\cdots \\
\left.\left\langle\bar{\psi} \bar{\psi}\left|\mathcal{O}_{B}\right| 0\right\rangle\right|_{\mathrm{UV}} & :=\langle 21\rangle a^{2}\left(\mu_{R}\right)\left(\frac{1}{g} \cdot k\right)+\cdots \\
\left.\left\langle\bar{X} \bar{Y} \bar{Z}\left|\mathcal{O}_{B}\right| 0\right\rangle\right|_{\mathrm{UV}} & :=b^{(1)} a\left(\mu_{R}\right)+b^{(2)} a^{2}\left(\mu_{R}\right)+\cdots
\end{aligned}
$$

where the coefficients carrying the UV divergences are

$$
\begin{aligned}
f^{(1)} & =\frac{f_{1}^{(1)}}{\epsilon}, & f^{(2)} & =\frac{f_{2}^{(2)}}{\epsilon^{2}}+\frac{f_{1}^{(2)}}{\epsilon}, \\
b^{(1)} & =\frac{b_{1}^{(1)}}{\epsilon}, & b^{(2)} & =\frac{b_{2}^{(2)}}{\epsilon^{2}}+\frac{b_{1}^{(2)}}{\epsilon}, \\
h & =\frac{h_{1}}{\epsilon}, & k & =\frac{k_{1}}{\epsilon},
\end{aligned}
$$

and the running 't Hooft coupling $a\left(\mu_{R}\right)$ defined in (2.14) counts the number of loops. We have also been careful in distinguishing the coupling constant $g$ from $a\left(\mu_{R}\right)$ on the righthand side of (7.3)-(7.6). Note that in (7.3) and (7.5) we have factored out the tree-level form factor $\left\langle 1^{\bar{\psi}} 2^{\bar{\psi}}\left|\frac{1}{2} \operatorname{Tr}(\psi \psi)\right| 0\right\rangle^{(0)}=\langle 21\rangle$.

A few comments on these expansions are in order.

1. We have performed explicit perturbative calculations in previous sections that allow us to extract (7.4)-(7.6), and we will shortly explain how to extract the UV-poles for (7.3).

2. (7.4) is the result of a one-loop calculation (hence the single power of $a\left(\mu_{R}\right)$ involving a five-point amplitude, which is $\mathcal{O}\left(g^{3}\right)$ (hence the extra power of $g$ ).

3. (7.5) is the result of a two-loop calculation, again involving a five-point amplitude. This is proportional to $a\left(\mu_{R}\right)^{2} / g$, which is $\mathcal{O}\left(g^{3}\right)$ just like (7.4).

Expanding the mixing matrix $\mathcal{Z}$ as

$$
\mathcal{Z}=\mathbb{1}+\sum_{L=1}^{\infty} \mathcal{Z}^{(L)}:=\mathbb{1}+\sum_{L=1}^{\infty} a\left(\mu_{R}\right)^{L} z^{(L)},
$$

and requiring the finiteness of the renormalised form factors we arrive at

$$
\begin{array}{rlrl}
\left(z^{(1)}\right)_{F} F & =-\frac{f_{1}^{(1)}}{\epsilon}, & \left(z^{(2)}\right)_{F}^{F}=-\frac{f_{2}^{(2)}-\left(f_{1}^{(1)}\right)^{2}}{\epsilon^{2}}-\frac{f_{1}^{(2)}}{\epsilon}, \\
\left(z^{(1)}\right)_{B}{ }^{B}=-\frac{b_{1}^{(1)}}{\epsilon}, & \left(z^{(2)}\right)_{B}{ }^{B}=-\frac{b_{2}^{(2)}-\left(b_{1}^{(1)}\right)^{2}}{\epsilon^{2}}-\frac{b_{1}^{(2)}}{\epsilon}, \\
\left(z^{(1)}\right)_{F}{ }^{B}=-g \cdot \frac{h_{1}}{\epsilon}, & \left(z^{(2)}\right)_{B}{ }^{F}=-\frac{1}{g} \cdot \frac{k_{1}}{\epsilon} .
\end{array}
$$


Note that from $(7.8) \mathcal{Z}^{(L)}:=a\left(\mu_{R}\right)^{L} z^{(L)}$. The $\log \mathcal{Z}$ matrix has the form, up to $\mathcal{O}\left(a\left(\mu_{R}\right)^{2}\right)$,

$$
\begin{aligned}
\log (\mathcal{Z}) & \sim\left(\begin{array}{cc}
\left(\mathcal{Z}^{(1)}\right)_{F}{ }^{F}+\left[\left(\mathcal{Z}^{(2)}\right)_{F}{ }^{F}-\frac{1}{2}\left(\left(\mathcal{Z}^{(1)}\right)_{F}{ }^{F}\right)^{2}\right] & \left(\mathcal{Z}^{(1)}\right)_{F}^{B} \\
\left(\mathcal{Z}^{(2)}\right)_{B}{ }^{F} & \left(\mathcal{Z}^{(1)}\right)_{B}{ }^{B}+\left[\left(\mathcal{Z}^{(2)}\right)_{B}{ }^{B}-\frac{1}{2}\left(\left(\mathcal{Z}^{(1)}\right)_{B}{ }^{B}\right)^{2}\right]
\end{array}\right) \\
= & \left(\begin{array}{cc}
-a\left(\mu_{R}\right) \frac{f_{1}^{(1)}}{\epsilon}-a^{2}\left(\mu_{R}\right) \frac{f_{2}^{(2)}-\frac{1}{2}\left(f_{1}^{(1)}\right)^{2}}{\epsilon^{2}} & -g a\left(\mu_{R}\right) \cdot \frac{h_{1}}{\epsilon} \\
-\frac{a\left(\mu_{R}\right)}{g} \cdot \frac{k_{1}}{\epsilon} & -a\left(\mu_{R}\right) \frac{b_{1}^{(1)}}{\epsilon}-a^{2}\left(\mu_{R}\right) \frac{b_{2}^{(2)}-\frac{1}{2}\left(b_{1}^{(1)}\right)^{2}}{\epsilon^{2}}
\end{array}\right) .
\end{aligned}
$$

We now move on to determine the various matrix elements. From (2.12) we read off that $b_{1}^{(1)}=-6$, and hence

$$
\left(z^{(1)}\right)_{B}^{B}=\frac{6}{\epsilon}
$$

Next we compute $\left(z^{(2)}\right)_{B}{ }^{B}-(1 / 2)\left(\left(z^{(1)}\right)_{B}{ }^{B}\right)^{2}$. This quantity has already been calculated in section 4 , and we remark that we should drop the $\pi^{2}$ term in (4.9), which is not of UV origin. Doing so we find $b_{2}^{(2)}-(1 / 2)\left(b_{1}^{(1)}\right)^{2}=18 / \epsilon$, and therefore

$$
\left(z^{(2)}\right)_{B}{ }^{B}-\frac{1}{2}\left(\left(z^{(1)}\right)_{B}^{B}\right)^{2}=-\frac{18}{\epsilon} .
$$

Importantly, the $1 / \epsilon^{2}$ pole is absent in (7.12). Next, from the two-loop result of (6.6) we obtain

$$
\left(z^{(2)}\right)_{B}^{F}=-\frac{6}{\epsilon} \cdot \frac{1}{g}
$$

while from (5.16) we find

$$
\left(z^{(1)}\right)_{F}^{B}=-\frac{6}{\epsilon} \cdot g
$$

Finally, we need to determine $\left(z^{(1)}\right)_{F}{ }^{F}$ and $\left(z^{(2)}\right)_{F}{ }^{F}$. In order to do so, we recall that $\mathcal{O}_{F}$ appears as a component of the chiral part of the stress tensor multiplet operator (see eq. (3.3) of [48]). Super form factors of this protected operator were first studied in [7]. The components of this multiplet can be obtained by acting with four of the eight supercharges $Q_{A \alpha}$ with $A=3,4$ on the bottom component $\operatorname{Tr}\left(X^{2}\right)=\operatorname{Tr}\left(\phi_{12}^{2}\right)$. Using the explicit supersymmetry transformation in eq. (A.15) of [48], adapted to our conventions, and acting with $Q_{3}^{\alpha} Q_{3 \alpha}$ on the bottom component we find the following half-BPS descendent of $\operatorname{Tr}\left(\phi_{12}^{2}\right)$,

$$
\mathcal{O}_{\mathrm{BPS}^{\prime}}:=\frac{1}{2} \operatorname{Tr}\left(\psi^{\alpha} \psi_{\alpha}\right)+g \operatorname{Tr}(X[Y, Z])=\mathcal{O}_{F}+g \mathcal{O}_{B}
$$


Since this operator is half-BPS the corresponding form factors are UV finite. Hence we infer that

$$
\left.F_{\mathcal{O}_{F}}\left(1^{\bar{\psi}^{123}}, 2^{\bar{\psi}^{123}} ; q\right)\right|_{\mathrm{UV}}=-\left.g F_{\mathcal{O}_{B}}\left(1^{\bar{\psi}^{123}}, 2^{\bar{\psi}^{123}} ; q\right)\right|_{\mathrm{UV}}
$$

from which we get

$$
\left(z^{(1)}\right)_{F}{ }^{F}=-g\left(z^{(1)}\right)_{B}{ }^{F}=0, \quad\left(z^{(2)}\right)_{F}{ }^{F}=-g\left(z^{(2)}\right)_{B}{ }^{F}=\frac{6}{\epsilon} .
$$

Using (7.16) we then obtain

$$
\left(z^{(2)}\right)_{F}^{F}-\frac{1}{2}\left(\left(z^{(1)}\right)_{F}^{F}\right)^{2}=\frac{6}{\epsilon}
$$

We can now write down the matrix (7.10), with the result

$$
\log (\mathcal{Z})=\left(\begin{array}{cc}
a^{2}\left(\mu_{R}\right) \frac{6}{\epsilon} & -a\left(\mu_{R}\right) g \frac{6}{\epsilon} \\
-\frac{a^{2}\left(\mu_{R}\right)}{g} \cdot \frac{6}{\epsilon} & a\left(\mu_{R}\right) \cdot \frac{6}{\epsilon}-a^{2}\left(\mu_{R}\right) \cdot \frac{18}{\epsilon}
\end{array}\right)+\mathcal{O}\left(a\left(\mu_{R}\right)^{3}\right)
$$

Finally, the dilatation operator up to two loops is

$$
\delta \mathfrak{D}=\lim _{\epsilon \rightarrow 0}\left[-\mu_{R} \frac{\partial}{\partial \mu_{R}} \log (\mathcal{Z})\right]=12 \times\left(\begin{array}{cc}
2 a^{2} & -a g \\
-2 \frac{a^{2}}{g} & a-6 a^{2}
\end{array}\right)
$$

where we recall that our 't Hooft coupling is defined in (2.18). The eigenvalues of this matrix are the anomalous dimensions of the eigenstates of the dilatation operator. One of them vanishes indicating the presence of a non-trivial additional protected operator. The second one is

$$
\gamma_{\mathcal{K}}=12 a-48 a^{2}+\mathcal{O}\left(a^{3}\right)
$$

in precise agreement with the one- and two-loop anomalous dimensions for the Konishi supermultiplet. We can also write the corresponding eigenstates by diagonalising the transpose of $\delta \mathfrak{D} .{ }^{14}$ One arrives at the two operators [35-38]

$$
\begin{aligned}
\mathcal{O}_{\mathrm{BPS}^{\prime}} & =\mathcal{O}_{F}+g \mathcal{O}_{B} \\
\mathcal{O}_{\mathcal{K}} & =\mathcal{O}_{B}-\frac{g N}{8 \pi^{2}} \mathcal{O}_{F} .
\end{aligned}
$$

The first one is the protected operator introduced in (7.15) above, while the second combination is a descendant of the Konishi operator.

\footnotetext{
${ }^{14}$ Note that in this sector $\delta \mathfrak{D}$ is not symmetric. A generic combination of the two operators $\mathcal{O}_{F}$ and $\mathcal{O}_{B}$ can be written as $v_{f} \mathcal{O}_{F}+v_{b} \mathcal{O}_{B}:=(\mathbf{v}, \mathbf{O})$, with $\mathbf{v}^{T}:=\left(v_{F}, v_{B}\right)$ and $\mathbf{O}^{T}:=\left(\mathcal{O}_{F}, \mathcal{O}_{B}\right)$. Under the action of the dilatation operator we have $(\mathbf{v}, \mathbf{O}) \rightarrow(\mathbf{v}, \delta \mathfrak{D} \mathbf{O})=\left((\delta \mathfrak{D})^{T} \mathbf{v}, \mathbf{O}\right)$.
} 


\section{Conclusions}

There are several natural continuations of the work presented in this paper. In particular, it would be interesting to consider wider classes of non-protected operators than those considered here and in $[30,49]$. Potentially this could lead to new insights and approaches to integrability. For example, [29] established a direct link between minimal one-loop form factors of general operators and Zwiebel's form of the one-loop dilatation operator [34]. In [50] it was shown, using this form of the dilatation operator, how the Yangian symmetry [51] of the tree-level $S$-matrix of $\mathcal{N}=4$ SYM implies the Yangian symmetry of the one-loop dilatation operator, which in turn is related to its integrability [52]. Clearly it would be very interesting to generalise this to higher loops.

In [7], supersymmetric Ward identities were used to relate form factors of all the different operators in the protected stress tensor multiplet to form factors of the chiral primary operator $\operatorname{Tr}\left(X^{2}\right)$ at any loop order. This led naturally to the definition of super form factors extending the Nair on-shell superspace used for amplitudes in $\mathcal{N}=4$ SYM. It would be interesting to extend this to non-protected operators contained in larger multiplets. Technically this is more challenging but first important steps in this direction have been taken in recent papers [53, 54] and [55] where tree-level MHV form factors for arbitrary unprotected operators were constructed using twistor actions and Lorentz harmonic chiral superspace, respectively.

It seems plausible that a more detailed study of minimal and slightly non-minimal two-loop form factors of non-protected operators will reveal a set of unique building blocks with different degrees of transcendentality for form factors of arbitrary operators. One piece of evidence is the equivalence of the two-loop, three-point form factor of $\operatorname{Tr}\left(X^{2}\right)$ and the maximally transcendental part of Higgs to three-gluon amplitudes [6]. It would be natural to expect that the universality of the leading transcendental part extends also to all length-two operators such as $\operatorname{Tr}(D F D F)$ in any non-abelian gauge theory. Another piece of evidence is that the minimal two-loop form factor of $\operatorname{Tr}\left(X^{3}\right)$ [26] equals the leading transcendentality part of the minimal two-loop form factors in the $\mathrm{SU}(2)$ sector [30] and in the $\mathrm{SU}(2 \mid 3)$ sector studied in the present paper. We would expect that this universality also applies to operators like $\operatorname{Tr}\left(F^{3}\right)$ in $\mathcal{N}=4$ SYM, and possibly also in QCD and pure YangMills. Furthermore the intriguing relation of terms of lower transcendentality appearing in the $\mathrm{SU}(2)$ and $\mathrm{SU}(2 \mid 3)$ sectors (see section 4) points at further unexpected regularities to be explored. We intend to return to these issues in the very near future.

\section{Acknowledgments}

We would like to thank Burkhard Eden, Joseph Hayling, Paul Heslop, Gregory Korchemsky, Paolo Mattioli, Costis Papageorgakis, Sanjaye Ramgoolam, Radu Roiban and Rodolfo Russo for useful discussions and comments. AB and GT would like to thank NORDITA and the organisers of the "Aspects of Amplitudes" programme for hospitality during the last stage of this work. MK is supported by an STFC studentship. Part of BP's work leading to these results was carried out during her PhD at the Centre for Research in String Theory 
of Queen Mary University of London. The work of BP is funded by the People Programme (Marie Curie Actions) of the European Union's Seventh Framework Programme FP7/20072013/ under REA Grant Agreement No 317089 (GATIS). This work was supported by the Science and Technology Facilities Council Consolidated Grant ST/L000415/1 "String theory, gauge theory \& duality".

\section{A One-loop integral functions}

Throughout the paper, we use the following conventions for the one-loop massless scalar integrals in dimensional regularisation (upper/lower-case letters correspond to massive/massless momenta) [56]:
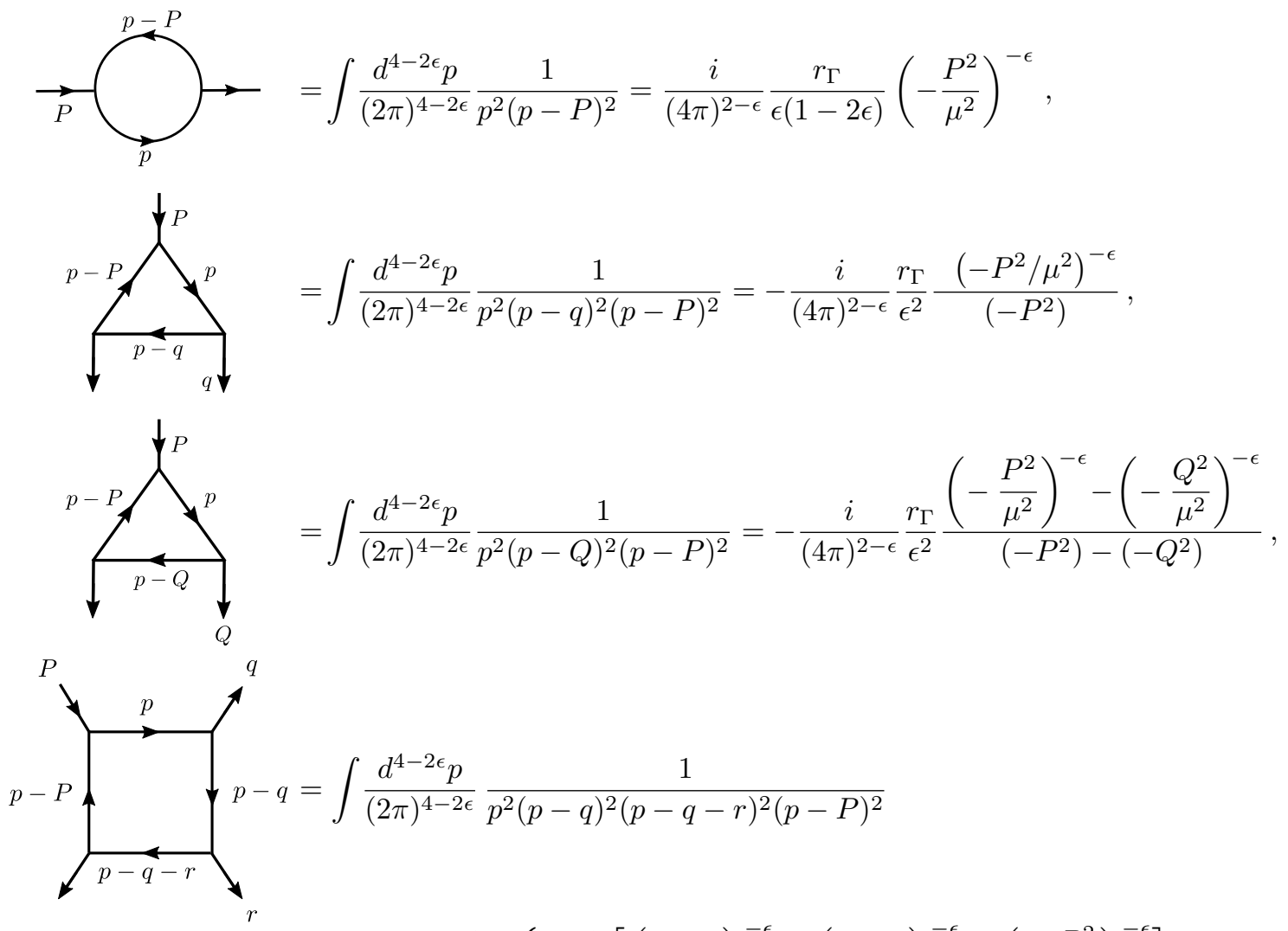

$$
\begin{aligned}
= & \int \frac{d^{4-2 \epsilon} p}{(2 \pi)^{4-2 \epsilon}} \frac{1}{p^{2}(p-q)^{2}(p-q-r)^{2}(p-P)^{2}} \\
= & -\frac{i}{(4 \pi)^{2-\epsilon}} \frac{2 r_{\Gamma}}{s t}\left\{-\frac{1}{\epsilon^{2}}\left[\left(-\frac{s}{\mu^{2}}\right)^{-\epsilon}+\left(-\frac{t}{\mu^{2}}\right)^{-\epsilon}-\left(-\frac{P^{2}}{\mu^{2}}\right)^{-\epsilon}\right]\right. \\
& \left.+\operatorname{Li}_{2}\left(1-\frac{P^{2}}{s}\right)+\operatorname{Li}_{2}\left(1-\frac{P^{2}}{t}\right)+\frac{1}{2} \log ^{2}\left(\frac{s}{t}\right)+\frac{\pi^{2}}{6}\right\}
\end{aligned}
$$

where

$$
r_{\Gamma}=\frac{\Gamma(1+\epsilon) \Gamma(1-\epsilon)^{2}}{\Gamma(1-2 \epsilon)} .
$$




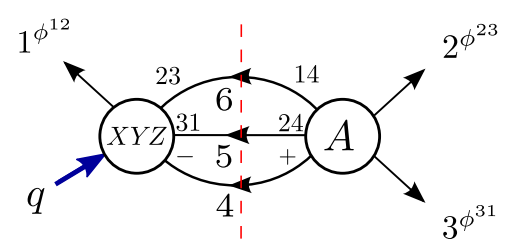

$(i)$

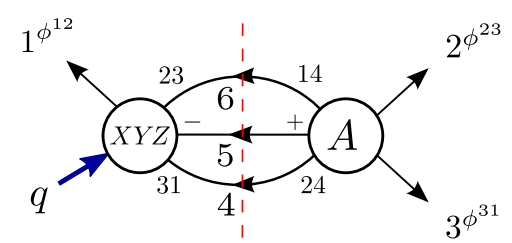

(ii)

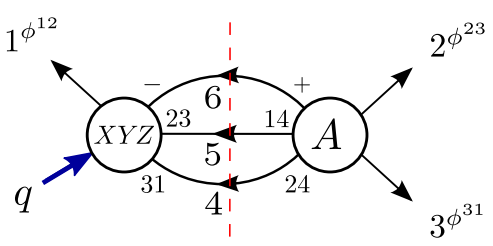

(iii)

Figure 17. Three possibilities for a single gluon running on one of the internal loop legs for $\operatorname{Tr}(X Y Z)$ operator.

\section{B Comparing half-BPS form factors}

In this appendix we present explicit calculations confirming that the minimal form factor of the half-BPS operator $\operatorname{Tr}(X\{Y, Z\})$ has the same integrand, and hence remainder, as that of the minimal form factor of $\operatorname{Tr}\left(X^{3}\right)$ considered in [26].

We begin by considering the three diagrams in the gluonic contribution to the $s_{23}-$ channel, presented in figure 17 below and corresponding to the $\operatorname{Tr}(X Y Z)$ operator. The corresponding integrands are

$$
\begin{gathered}
(i): i^{3} A\left(2^{\phi^{23}}, 3^{\phi^{31}}, 4^{+}, 5^{\phi^{24}}, 6^{\phi^{14}}\right) \times F_{\operatorname{Tr}(X Y Z)}^{(0)}\left(1^{\phi^{12}}, 6^{\phi^{23}}, 5^{\phi^{31}}, 4^{-} ; q\right)=-\frac{\langle 25\rangle\langle 36\rangle[51]}{\langle 34\rangle\langle 45\rangle\langle 62\rangle[54][41]}, \\
(i i): i^{3} A\left(2^{\phi^{23}}, 3^{\phi^{31}}, 4^{\phi^{24}}, 5^{+}, 6^{\phi^{14}}\right) \times F_{\operatorname{Tr}(X Y Z)}^{(0)}\left(1^{\phi^{12}}, 6^{\phi^{23}}, 5^{-}, 4^{\phi^{31}} ; q\right)=-\frac{\langle 24\rangle\langle 36\rangle\langle 46\rangle[64]}{\langle 34\rangle\langle 45\rangle\langle 56\rangle\langle 62\rangle[54][65]}, \\
(\text { iii }): i^{3} A\left(2^{\phi^{23}}, 3^{\phi^{31}}, 4^{\phi^{24}}, 5^{\phi^{14}}, 6^{+}\right) \times F_{\operatorname{Tr}(X Y Z)}^{(0)}\left(1^{\phi^{12}}, 6^{-}, 5^{\phi^{23}}, 4^{\phi^{33}} ; q\right)=-\frac{\langle 24\rangle\langle 35\rangle[15]}{\langle 34\rangle\langle 56\rangle\langle 62\rangle[16][65]} .
\end{gathered}
$$

We will combine these into "anti-commutator" pieces by appropriately adding to them $-1 / 2$ of the terms that appear in expressions (3.20)-(3.22), corresponding to the $\operatorname{Tr}(X Z Y)$ operator (removing the factor of -2 ). We then find for the diagrams in figure $8(i)$ and $17(i)$,

$$
\mathrm{AC}_{1}=-\frac{(\langle 25\rangle\langle 36\rangle+\langle 35\rangle\langle 62\rangle)[51]}{\langle 34\rangle\langle 45\rangle\langle 62\rangle[54][41]}=-\frac{\langle 23\rangle\langle 56\rangle[51]}{\langle 34\rangle\langle 45\rangle\langle 62\rangle[54][41]}
$$

Similarly, we find for the diagrams in figure 8(ii) and 17(ii),

$$
\mathrm{AC}_{2}=-\frac{\langle 23\rangle\langle 46\rangle\langle 46\rangle[64]}{\langle 34\rangle\langle 45\rangle\langle 56\rangle\langle 62\rangle[54][65]}
$$

and finally, for the integrands of figure $8(i i i)$ and $17(i i i)$,

$$
\mathrm{AC}_{3}=-\frac{\langle 23\rangle\langle 45\rangle[15]}{\langle 34\rangle\langle 56\rangle\langle 62\rangle[16][65]}
$$




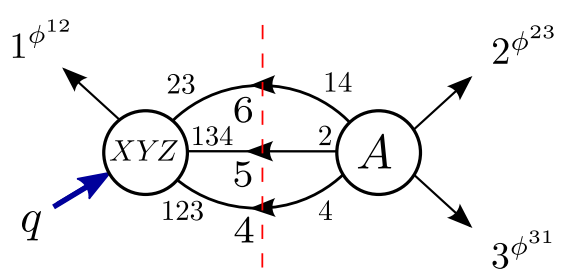

(i)

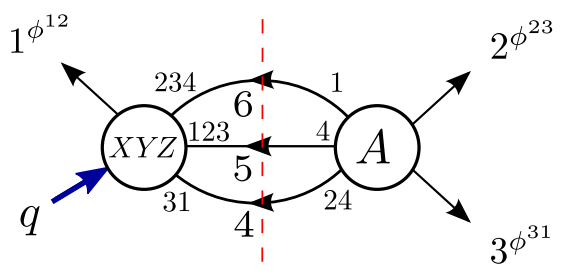

(iii)

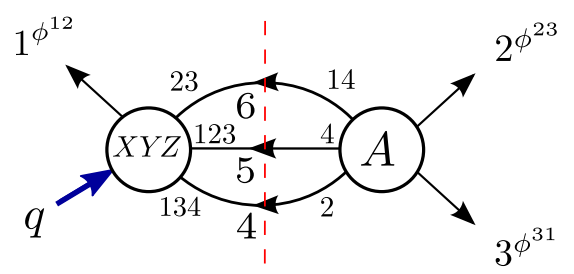

(ii)

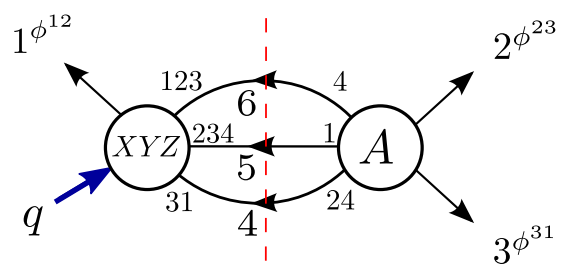

$(i v)$

Figure 18. Four possibilities for fermions running on the internal loop legs for the $\operatorname{Tr}(X Y Z)$ operator.

Next we consider the fermionic contributions to this cut for the operator $\operatorname{Tr}(X Y Z)$. These are presented in figure 18 below. The corresponding integrands are:

$$
\begin{gathered}
(i): i^{3} A\left(2^{\phi^{23}}, 3^{\phi^{31}}, 4^{\psi^{4}}, 5^{\psi^{2}}, 6^{\phi^{14}}\right) \times F_{\operatorname{Tr}(X Y Z)}^{(0)}\left(1^{\phi^{12}}, 6^{\phi^{23}}, 5^{\psi^{134}}, 4^{\bar{\psi}^{123}} ; q\right)=\frac{\langle 25\rangle\langle 46\rangle\langle 36\rangle}{\langle 34\rangle\langle 56\rangle\langle 62\rangle s_{45}}, \\
(i i): i^{3} A\left(2^{\phi^{23}}, 3^{\phi^{31}}, 4^{\psi^{4}}, 5^{\psi^{2}}, 6^{\phi^{14}}\right) \times F_{\operatorname{Tr}(X Y Z)}^{(0)}\left(1^{\phi^{12}}, 6^{\phi^{23}}, 5^{\bar{\psi}^{123}}, 4^{\psi^{134}} ; q\right)=-\frac{\langle 42\rangle\langle 36\rangle}{\langle 34\rangle\langle 62\rangle s_{45}}, \\
(i i i): i^{3} A\left(2^{\phi^{23}}, 3^{\phi^{31}}, 4^{\phi^{24}}, 5^{\psi^{4}}, 6^{\psi^{1}}\right) \times F_{\operatorname{Tr}(X Y Z)}^{(0)}\left(1^{\phi^{12}}, 6^{\psi^{234}}, 5^{\bar{\psi}^{123}}, 4^{\phi^{31}} ; q\right)=-\frac{\langle 36\rangle\langle 42\rangle}{\langle 34\rangle\langle 62\rangle s_{56}}, \\
(i v): i^{3} A\left(2^{\phi^{23}}, 3^{\phi^{31}}, 4^{\phi^{24}}, 5^{\psi^{1}}, 6^{\psi^{4}}\right) \times F_{\operatorname{Tr}(X Y Z)}^{(0)}\left(1^{\phi^{12}}, 6^{\bar{\psi}^{123}}, 5^{\psi^{234}}, 4^{\phi^{31}} ; q\right)=\frac{\langle 24\rangle\langle 35\rangle\langle 46\rangle}{\langle 34\rangle\langle 62\rangle\langle 45\rangle s_{56}} .
\end{gathered}
$$

We combine them similarly to the gluonic case: for the commutator, diagrams of figure 9 and 10 should come with an overall minus sign. After some algebra we find, for figure 9 plus figure $18(i)$ and (ii),

$$
\begin{aligned}
\mathrm{AC}_{4} & =\frac{1}{\langle 34\rangle\langle 56\rangle\langle 62\rangle s_{45}}(\langle 25\rangle\langle 36\rangle\langle 46\rangle-\langle 36\rangle\langle 42\rangle\langle 56\rangle-\langle 26\rangle\langle 35\rangle\langle 46\rangle+\langle 34\rangle\langle 56\rangle\langle 62\rangle) \\
& =-\frac{\langle 23\rangle}{\langle 34\rangle\langle 45\rangle\langle 56\rangle\langle 62\rangle} \frac{2\langle 46\rangle\langle 65\rangle}{[54]}
\end{aligned}
$$

and for figure 10 plus figure 18 (iii) and (iv),

$$
\begin{aligned}
\mathrm{AC}_{5} & =-\frac{1}{\langle 34\rangle\langle 45\rangle\langle 62\rangle s_{56}}(\langle 36\rangle\langle 42\rangle\langle 45\rangle-\langle 24\rangle\langle 35\rangle\langle 46\rangle+\langle 25\rangle\langle 46\rangle\langle 34\rangle-\langle 34\rangle\langle 45\rangle\langle 62\rangle) \\
& =-\frac{\langle 23\rangle}{\langle 34\rangle\langle 45\rangle\langle 56\rangle\langle 62\rangle} \frac{2\langle 45\rangle\langle 64\rangle}{[65]} .
\end{aligned}
$$


Finally we combine all the "anti-commutator" terms. After some manipulation, we get

$$
\begin{aligned}
\sum_{i=1}^{5} \mathrm{AC}_{i}=-\frac{\langle 23\rangle}{\langle 34\rangle\langle 45\rangle\langle 56\rangle\langle 62\rangle}[ & \frac{[51]\langle 54\rangle^{2}}{[65][16]}-2 \frac{\langle 54\rangle\langle 64\rangle}{[65]}+\frac{[16]\langle 64\rangle^{2}}{[65][51]} \\
& \left.-\frac{[14]\langle 46\rangle^{2}}{[45][51]}+2 \frac{\langle 46\rangle\langle 56\rangle}{[45]}-\frac{[51]\langle 56\rangle^{2}}{[45][14]}\right],
\end{aligned}
$$

which is precisely the result of the $s_{23}$-channel cut of operator $\operatorname{Tr}\left(X^{3}\right)$ as presented in eq. (3.16) of [26].

Open Access. This article is distributed under the terms of the Creative Commons Attribution License (CC-BY 4.0), which permits any use, distribution and reproduction in any medium, provided the original author(s) and source are credited.

\section{References}

[1] W.L. van Neerven, Infrared Behavior of On-shell Form-factors in a $N=4$ Supersymmetric Yang-Mills Field Theory, Z. Phys. C 30 (1986) 595 [InSPIRE].

[2] L.F. Alday and J. Maldacena, Comments on gluon scattering amplitudes via AdS/CFT, JHEP 11 (2007) 068 [arXiv:0710.1060] [InSPIRE].

[3] A. Brandhuber, B. Spence, G. Travaglini and G. Yang, Form Factors in $N=4$ Super Yang-Mills and Periodic Wilson Loops, JHEP 01 (2011) 134 [arXiv:1011.1899] [InSPIRE].

[4] T. Gehrmann, J.M. Henn and T. Huber, The three-loop form factor in $N=4$ super Yang-Mills, JHEP 03 (2012) 101 [arXiv:1112.4524] [INSPIRE].

[5] R. Boels, B.A. Kniehl and G. Yang, Master integrals for the four-loop Sudakov form factor, Nucl. Phys. B 902 (2016) 387 [arXiv: 1508.03717] [INSPIRE].

[6] A. Brandhuber, G. Travaglini and G. Yang, Analytic two-loop form factors in $N=4 S Y M$, JHEP 05 (2012) 082 [arXiv: 1201.4170] [INSPIRE].

[7] A. Brandhuber, O. Gurdogan, R. Mooney, G. Travaglini and G. Yang, Harmony of Super Form Factors, JHEP 10 (2011) 046 [arXiv:1107.5067] [INSPIRE].

[8] L.V. Bork, D.I. Kazakov and G.S. Vartanov, On MHV Form Factors in Superspace for $\backslash=4$ SYM Theory, JHEP 10 (2011) 133 [arXiv:1107.5551] [INSPIRE].

[9] F. Wilczek, Decays of Heavy Vector Mesons Into Higgs Particles, Phys. Rev. Lett. 39 (1977) 1304 [INSPIRE].

[10] M.A. Shifman, A.I. Vainshtein, M.B. Voloshin and V.I. Zakharov, Low-Energy Theorems for Higgs Boson Couplings to Photons, Sov. J. Nucl. Phys. 30 (1979) 711 [Yad. Fiz. 30 (1979) 1368] [INSPIRE].

[11] S. Dawson, Radiative corrections to Higgs boson production, Nucl. Phys. B 359 (1991) 283 [INSPIRE].

[12] M. Spira, A. Djouadi, D. Graudenz and P.M. Zerwas, Higgs boson production at the LHC, Nucl. Phys. B 453 (1995) 17 [hep-ph/9504378] [INSPIRE].

[13] L.J. Dixon, E.W.N. Glover and V.V. Khoze, MHV rules for Higgs plus multi-gluon amplitudes, JHEP 12 (2004) 015 [hep-th/0411092] [INSPIRE]. 
[14] S.D. Badger, E.W.N. Glover and V.V. Khoze, MHV rules for Higgs plus multi-parton amplitudes, JHEP 03 (2005) 023 [hep-th/0412275] [INSPIRE].

[15] S.D. Badger and E.W.N. Glover, One-loop helicity amplitudes for $H \rightarrow$ gluons: The All-minus configuration, Nucl. Phys. Proc. Suppl. 160 (2006) 71 [hep-ph/0607139] [INSPIRE].

[16] S.D. Badger, E.W.N. Glover and K. Risager, One-loop phi-MHV amplitudes using the unitarity bootstrap, JHEP 07 (2007) 066 [arXiv:0704.3914] [INSPIRE].

[17] S. Badger, E.W. Nigel Glover, P. Mastrolia and C. Williams, One-loop Higgs plus four gluon amplitudes: Full analytic results, JHEP 01 (2010) 036 [arXiv:0909.4475] [INSPIRE].

[18] T. Gehrmann, M. Jaquier, E.W.N. Glover and A. Koukoutsakis, Two-Loop QCD Corrections to the Helicity Amplitudes for $H \rightarrow 3$ partons, JHEP 02 (2012) 056 [arXiv:1112.3554] [INSPIRE].

[19] A. Koukoutsakis, Higgs bosons and QCD jets at two loops, Ph.D. Thesis, Durham University, Durham U.K. (2003).

[20] D. Neill, Two-Loop Matching onto Dimension Eight Operators in the Higgs-Glue Sector, arXiv:0908.1573 [INSPIRE].

[21] S. Dawson, I.M. Lewis and M. Zeng, Effective field theory for Higgs boson plus jet production, Phys. Rev. D 90 (2014) 093007 [arXiv: 1409.6299] [INSPIRE].

[22] J.A. Gracey, Classification and one loop renormalization of dimension-six and dimension-eight operators in quantum gluodynamics, Nucl. Phys. B 634 (2002) 192 [Erratum ibid. B 696 (2004) 295] [hep-ph/0204266] [INSPIRE].

[23] L.J. Dixon and Y. Shadmi, Testing gluon selfinteractions in three jet events at hadron colliders, Nucl. Phys. B 423 (1994) 3 [hep-ph/9312363] [INSPIRE].

[24] J. Broedel and L.J. Dixon, Color-kinematics duality and double-copy construction for amplitudes from higher-dimension operators, JHEP 10 (2012) 091 [arXiv:1208.0876] [INSPIRE].

[25] B. Penante, B. Spence, G. Travaglini and C. Wen, On super form factors of half-BPS operators in $N=4$ super Yang-Mills, JHEP 04 (2014) 083 [arXiv:1402.1300] [INSPIRE].

[26] A. Brandhuber, B. Penante, G. Travaglini and C. Wen, The last of the simple remainders, JHEP 08 (2014) 100 [arXiv:1406.1443] [INSPIRE].

[27] N. Beisert, The complete one loop dilatation operator of $N=4$ super Yang-Mills theory, Nucl. Phys. B 676 (2004) 3 [hep-th/0307015] [INSPIRE].

[28] N. Beisert, The SU(2|3) dynamic spin chain, Nucl. Phys. B 682 (2004) 487 [hep-th/0310252] [INSPIRE].

[29] M. Wilhelm, Amplitudes, Form Factors and the Dilatation Operator in $\mathcal{N}=4$ SYM Theory, JHEP 02 (2015) 149 [arXiv: 1410.6309] [INSPIRE].

[30] F. Loebbert, D. Nandan, C. Sieg, M. Wilhelm and G. Yang, On-Shell Methods for the Two-Loop Dilatation Operator and Finite Remainders, JHEP 10 (2015) 012 [arXiv: 1504.06323] [INSPIRE].

[31] L. Koster, V. Mitev and M. Staudacher, A Twistorial Approach to Integrability in $\mathcal{N}=4$ SYM, Fortsch. Phys. 63 (2015) 142 [arXiv:1410.6310] [INSPIRE].

[32] A. Brandhuber, B. Penante, G. Travaglini and D. Young, Integrability and unitarity, JHEP 05 (2015) 005 [arXiv: 1502.06627] [INSPIRE]. 
[33] A. Brandhuber, B. Penante, G. Travaglini and D. Young, Integrability and MHV diagrams in $N=4$ supersymmetric Yang-Mills theory, Phys. Rev. Lett. 114 (2015) 071602 [arXiv:1412.1019] [INSPIRE].

[34] B.I. Zwiebel, From Scattering Amplitudes to the Dilatation Generator in $N=4 S Y M, J$. Phys. A 45 (2012) 115401 [arXiv:1111.0083] [InSPIRE].

[35] K.A. Intriligator and W. Skiba, Bonus symmetry and the operator product expansion of $N=4$ Super Yang-Mills, Nucl. Phys. B 559 (1999) 165 [hep-th/9905020] [INSPIRE].

[36] M. Bianchi, S. Kovacs, G. Rossi and Y.S. Stanev, Properties of the Konishi multiplet in $N=4$ SYM theory, JHEP 05 (2001) 042 [hep-th/0104016] [INSPIRE].

[37] B. Eden, C. Jarczak, E. Sokatchev and Ya. S. Stanev, Operator mixing in $N=4$ SYM: The Konishi anomaly revisited, Nucl. Phys. B 722 (2005) 119 [hep-th/0501077] [INSPIRE].

[38] B. Eden, Operator mixing in N=4 SYM: The Konishi anomaly re-re-visited, Nucl. Phys. B 843 (2011) 223 [arXiv:0911.4884] [INSPIRE].

[39] Z. Bern, L.J. Dixon, D.C. Dunbar and D.A. Kosower, One loop $n$ point gauge theory amplitudes, unitarity and collinear limits, Nucl. Phys. B 425 (1994) 217 [hep-ph/9403226] [INSPIRE].

[40] J.J.M. Carrasco, Gauge and Gravity Amplitude Relations, arXiv:1506.00974 [INSPIRE].

[41] T. Gehrmann and E. Remiddi, Differential equations for two loop four point functions, Nucl. Phys. B 580 (2000) 485 [hep-ph/9912329] [INSPIRE].

[42] T. Gehrmann and E. Remiddi, Two loop master integrals for $\gamma^{*} \rightarrow 3$ jets: The Planar topologies, Nucl. Phys. B 601 (2001) 248 [hep-ph/0008287] [INSPIRE].

[43] R.N. Lee, Presenting LiteRed: a tool for the Loop InTEgrals REDuction, arXiv:1212.2685 [INSPIRE].

[44] R.N. Lee, LiteRed 1.4: a powerful tool for reduction of multiloop integrals, J. Phys. Conf. Ser. 523 (2014) 012059 [arXiv: 1310.1145] [INSPIRE].

[45] C. Anastasiou, Z. Bern, L.J. Dixon and D.A. Kosower, Planar amplitudes in maximally supersymmetric Yang-Mills theory, Phys. Rev. Lett. 91 (2003) 251602 [hep-th/0309040] [INSPIRE].

[46] Z. Bern, L.J. Dixon and V.A. Smirnov, Iteration of planar amplitudes in maximally supersymmetric Yang-Mills theory at three loops and beyond, Phys. Rev. D 72 (2005) 085001 [hep-th/0505205] [INSPIRE].

[47] A.B. Goncharov, M. Spradlin, C. Vergu and A. Volovich, Classical Polylogarithms for Amplitudes and Wilson Loops, Phys. Rev. Lett. 105 (2010) 151605 [arXiv:1006.5703] [INSPIRE].

[48] B. Eden, P. Heslop, G.P. Korchemsky and E. Sokatchev, The super-correlator/super-amplitude duality: Part I, Nucl. Phys. B 869 (2013) 329 [arXiv:1103.3714] [INSPIRE].

[49] D. Nandan, C. Sieg, M. Wilhelm and G. Yang, Cutting through form factors and cross sections of non-protected operators in $\mathcal{N}=4$ SYM, JHEP 06 (2015) 156 [arXiv:1410.8485] [INSPIRE]. 
[50] A. Brandhuber, P. Heslop, G. Travaglini and D. Young, Yangian Symmetry of Scattering Amplitudes and the Dilatation Operator in $N=4$ Supersymmetric Yang-Mills Theory, Phys. Rev. Lett. 115 (2015) 141602 [arXiv:1507.01504] [INSPIRE].

[51] J.M. Drummond, J.M. Henn and J. Plefka, Yangian symmetry of scattering amplitudes in $N=4$ super Yang-Mills theory, JHEP 05 (2009) 046 [arXiv:0902.2987] [INSPIRE].

[52] L. Dolan, C.R. Nappi and E. Witten, A Relation between approaches to integrability in superconformal Yang-Mills theory, JHEP 10 (2003) 017 [hep-th/0308089] [INSPIRE].

[53] L. Koster, V. Mitev, M. Staudacher and M. Wilhelm, Composite Operators in the Twistor Formulation of $\mathcal{N}=4$ Supersymmetric Yang-Mills Theory, Phys. Rev. Lett. 117 (2016) 011601 [arXiv: 1603.04471] [INSPIRE].

[54] L. Koster, V. Mitev, M. Staudacher and M. Wilhelm, All Tree-Level MHV Form Factors in $\mathcal{N}=4$ SYM from Twistor Space, JHEP 06 (2016) 162 [arXiv: 1604.00012] [INSPIRE].

[55] D. Chicherin and E. Sokatchev, Composite operators and form factors in $N=4 S Y M$, arXiv: 1605.01386 [INSPIRE].

[56] Z. Bern, L.J. Dixon, D.C. Dunbar and D.A. Kosower, Fusing gauge theory tree amplitudes into loop amplitudes, Nucl. Phys. B 435 (1995) 59 [hep-ph/9409265] [INSPIRE]. 